IOS Press

\title{
The Nondeterministic Information Logic NIL is PSPACE-complete
}

\author{
Stéphane Demri \\ Laboratoire LEIBNIZ-C.N.R.S. \\ 46 Avenue Félix Viallet \\ 38031 Grenoble, France. \\ email: demri@imag.fr
}

\begin{abstract}
The nondeterministic information logic NIL has been introduced by Orłowska and Pawlak in 1984 as a logic for reasoning about total information systems with the similarity, the forward inclusion and the backward inclusion relations. In 1987, Vakarelov provides the first first-order characterization of structures derived from information systems and this has been done with the semantical structures of NIL. Since then, various extensions of NIL have been introduced and many issues for information logics about decidability and Hilbertstyle proof systems have been solved. However, computational complexity issues have been seldom attacked in the literature mainly because the information logics are propositional polymodal logics with interdependent modal connectives. We show that NIL satisfiability is a PSPACE-complete problem. PSPACE-hardness is shown to be an easy consequence of PSPACE-hardness of the well-known modal logic S4. The main difficulty is to show that NIL satisfiability is in PSPACE. To do so we present an original construction that extends various previous works by Ladner (1977), Halpern and Moses (1992) and Spaan (1993).
\end{abstract}

Keywords: information system, multimodal logic, computational complexity, Ladner-like algorithm.

\section{Introduction}

Information systems. The information logics derived from Pawlak's information systems [28] are intended to be able to reason about information systems. An information system can be seen as a structure $\langle O B, A T\rangle$ such that $O B$ is a nonempty set of objects, $A T$ is a nonempty set of attributes and each attribute $a \in A T$, is a mapping $a: O B \rightarrow \mathcal{P}\left(V_{a l}\right) \backslash\{\emptyset\}$ where $V a l_{a}$ is a nonempty set of values. For each object $x$ and for each attribute $a, a(x)$ can be read as 
the set of possible values of the attribute $a$ for the object $x$. In that setting, various derived relations between objects can be defined. We recall some of them below from the literature (see e.g. [23]). For any $x_{1}, x_{2} \in O B, A \subseteq A T$,

- $x_{1} \operatorname{ind}(A) x_{2} \stackrel{\text { def }}{\Leftrightarrow}$ for any $a \in A, a\left(x_{1}\right)=a\left(x_{2}\right)$ (indiscernibility) ;

- $x_{1} \operatorname{comp}(A) x_{2} \stackrel{\text { def }}{\Leftrightarrow}$ for any $a \in A, a\left(x_{1}\right)=V a l_{a} \backslash a\left(x_{2}\right)$ (complementarity);

- $x_{1} \operatorname{fin}(A) x_{2} \stackrel{\text { def }}{\Leftrightarrow}$ for any $a \in A, a\left(x_{1}\right) \subseteq a\left(x_{2}\right)$ (forward inclusion);

- $x_{1} \operatorname{bin}(A) x_{2} \stackrel{\text { def }}{\Leftrightarrow}$ for any $a \in A, a\left(x_{2}\right) \subseteq a\left(x_{1}\right)$ (backward inclusion);

- $x_{1} \operatorname{sim}(A) x_{2} \stackrel{\text { def }}{\Leftrightarrow}$ for any $a \in A, a\left(x_{1}\right) \cap a\left(x_{2}\right) \neq \emptyset$ (similarity).

$x_{1} \operatorname{ind}(A) x_{2}$ can be read as follows: the objects $x_{1}$ and $x_{2}$ cannot be distinguished modulo the set of attributes $A$. Similarly, $x_{1} \operatorname{sim}(A) x_{2}$ iff $x_{1}$ and $x_{2}$ are similar modulo $A$. The other relations $\operatorname{comp}(A), \operatorname{fin}(A)$ and $\operatorname{bin}(A)$ admit a reading in the same vein. The polymodal logics obtained from the information systems are multimodal logics such that the relations in the Kripke-style semantical structures correspond to relations between objects in the underlying information systems. The first information logic has been introduced in [24] and many others appeared later (see e.g. [11, 33, 1, 18, 34, 8]). The information logic NIL (introduced in [25]) is remarkable among the class of information logics. Indeed, in 1987, Vakarelov [32] provides the first first-order characterization of structures derived from information systems and this has been done with the semantical structures of NIL. The NIL semantical structures contain not only forward and backward inclusions derived relations but also the similarity relation. More precisely, the NIL frames are all the structures $\langle O B, \operatorname{fin}(A T), \operatorname{bin}(A T), \operatorname{sim}(A T)\rangle$ derived from some information system $\langle O B, A T\rangle$. Actually, in [32], it has been shown that in order to define NIL an additional condition (not present in [25]) between forward inclusion and similarity needs to be taken into account (the forthcoming condition (N4)).

Our contribution. The main contribution of the paper is to fully characterize the computational complexity of NIL satisfiability. Actually, we show that NIL satisfiability is a PSPACEcomplete problem (see e.g. [27] for a thorough introduction to complexity theory). So, NIL satisfiability captures the difficulty of the whole complexity class PSPACE that is the class of (decision) problems that can be solved by a deterministic Turing machine in polynomial space in the length of the input string. PSPACE-hardness with respect to logarithmic space transformations is shown to be an easy consequence of PSPACE-hardness of the well-known modal logic S4 [20]. The main difficulty is to show that NIL satisfiability is in PSPACE. To do so we present an original construction that extends various previous works in $[20,15,31]$. The technique can be extended to other information logics (and polymodal logics with interdependent modal connectives) and this shall be the topic of forthcoming papers. Moreover, the analysis developed in the paper could be easily plug into a labelled tableaux calculus for NIL. Such a calculus is not difficult to define following for instance $[2,7,35]$. 
Related work. The procedure designed in this paper has a direct filiation with the works of Ladner [20], Halpern and Moses [15] and Spaan [31]. Indeed, we shall use a tableau-based procedure to show that we do not need more than polynomial space to check NIL satisfiability. We cannot take advantage of [16] where the complexity of join modal logics is characterized (NIL contains interdependent modal connectives). The detection of cycles for S4 modalities in NIL is similar to the proof-theoretical results from [5] that is related to the techniques from $[20,14]$. Other proof-theoretical analysis about complexity issues for modal logics can be found in $[22,35]$.

\section{Definition of NIL and PSPACE-hardness}

For any set $X$, we write $X^{*}$ [resp. $X^{+}$] to denote the set of [resp. nonempty] finite strings built from elements of $X$. For any finite string $s$, we write $|s|$ [resp. last $(s)$ ] to denote its length [resp. the last element of $s$, if any]. For any $s \in X^{*}$, we write $s^{k}$ to denote the string composed of $k$ copies of $s$. For instance, $(\text { bin } \cdot \text { fin })^{2}=$ bin $\cdot$ fin $\cdot$ bin $\cdot$ fin and $\mid(\text { bin } \cdot \text { fin })^{2} \mid=4$.

Given a countably infinite set For $_{0}=\left\{\mathrm{p}_{0}, \mathrm{p}_{1}, \mathrm{p}_{2}, \ldots\right\}$ of propositional variables the NILformulae $\phi$ are inductively defined as follows:

$$
\phi::=\mathrm{p}_{k}\left|\phi_{1} \wedge \phi_{2}\right| \neg \phi|[\leq] \phi|[\geq] \phi \mid[\sigma] \phi
$$

for $\mathrm{p}_{k} \in$ For $_{0}$. We write $|\phi|$ to denote the length of the formula $\phi$, that is the length of the string $\phi([\geq],[\leq]$ and $[\sigma]$ count here for one symbol). We write $\operatorname{md}(\phi)$ to denote the modal degree of $\phi$, that is the modal depth of $\phi$. md is naturally extended to finite sets of formulae, understood as conjunctions and by convention $\operatorname{md}(\emptyset)=0$. For $\mathrm{R} \in\{\sigma, \leq, \geq\},[\mathrm{R}]^{i} \phi$ is inductively defined as follows: $[\mathrm{R}]^{0} \phi=\phi$ and $[\mathrm{R}]^{i+1} \phi=[\mathrm{R}][\mathrm{R}]^{i} \phi$ for $i \geq 1$. For $s \in\{[\leq],[\geq],[\sigma]\}^{*}$, an $s$-formula is defined as a formula prefixed by $s$. For instance, $[\sigma][\leq][\geq] \mathrm{p}_{0}$ is a $[\sigma][\leq]$-formula. A NIL-model $\mathcal{M}$ is a structure $\mathcal{M}=\left\langle W, R_{\leq}, R_{\geq}, R_{\sigma}, m\right\rangle$ such that $W$ is a nonempty set, $m$ assigns to each propositional variable a subset of $W$ and $R_{\leq}, R_{\geq}, R_{\sigma}$ are binary relations on $W$ such that

(N1) $R_{\leq}=\left(R_{\geq}\right)^{-1}$, that is $R_{\leq}$is the converse of $R_{\geq}$;

(N2) $R_{\leq}$is reflexive and transitive;

(N3) $R_{\sigma}$ is reflexive and symmetric;

(N4) If $\langle x, y\rangle \in R_{\sigma},\left\langle x, x^{\prime}\right\rangle \in R_{\leq}$and $\left\langle y, y^{\prime}\right\rangle \in R_{\leq}$, then $\left\langle x^{\prime}, y^{\prime}\right\rangle \in R_{\sigma}$.

Observe that in any NIL-model, we have $\left(R_{\leq} \cup R_{\geq}\right) \subseteq R_{\sigma}$. The condition (N4) was not originally in the logic NIL defined in [25] but it appeared in [32] for the first-order characterization of the structures

$$
\langle O B, \operatorname{fin}(A T), \operatorname{bin}(A T), \operatorname{sim}(A T)\rangle
$$

where $\langle O B, A T\rangle$ is an information system. The condition (N4) is mainly responsible for the difficulty in showing that NIL satisfiability is in PSPACE. Conditions (N2) and (N4) can be equivalently replaced by

$\left(\mathrm{N}^{\prime}\right) R_{\geq}$is reflexive and transitive; 
$\left(\mathrm{N} 4^{\prime}\right)$ If $\langle x, y\rangle \in R_{\sigma}$ and $\left\langle y, y^{\prime}\right\rangle \in R_{\leq}$, then $\left\langle x, y^{\prime}\right\rangle \in R_{\sigma}$.

The condition $\left(\mathrm{N} 4^{\prime}\right)$ can be read as a pseudo transitivity condition involving two binary relations. Transitivity of $R_{\leq}$and $R_{\geq}$requires a specific treatment in order to show that NIL-satisfiability is in PSPACE. We apply a method of detection of cycles known in the literature (see $[20,31]$ ).

As is usual for modal logics, the formula $\phi$ is satisfied by the world $w \in W$ in $\mathcal{M} \stackrel{\text { def }}{\Leftrightarrow}$ $\mathcal{M}, w \models \phi$ where the satisfaction relation $\models$ is inductively defined as follows:

- $\mathcal{M}, w \models \mathrm{p} \stackrel{\text { def }}{\Leftrightarrow} w \in m(\mathrm{p})$, for every propositional variable $\mathrm{p}$;

- $\mathcal{M}, w \models[\leq] \phi \stackrel{\text { def }}{\Leftrightarrow}$ for every $w^{\prime} \in R_{\leq}(w), \mathcal{M}, w^{\prime}=\phi$;

- $\mathcal{M}, w \models[\geq] \phi \stackrel{\text { def }}{\Leftrightarrow}$ for every $w^{\prime} \in R_{\geq}(w), \mathcal{M}, w^{\prime}=\phi$;

- $\mathcal{M}, w \models[\sigma] \phi \stackrel{\text { def }}{\Leftrightarrow}$ for every $w^{\prime} \in R_{\sigma}(w), \mathcal{M}, w^{\prime} \models \phi$.

We omit the standard conditions for the propositional connectives. A formula $\phi$ is said to be NIL-satisfiable $\stackrel{\text { def }}{\Leftrightarrow}$ there is a NIL-model $\mathcal{M}=\left\langle W, R_{\leq}, R_{\geq}, R_{\sigma}, m\right\rangle$ and $w \in W$ such that $\mathcal{M}, w \models \phi$. An Hilbert-style system can be easily designed for NIL in which all the modal axiom schemes are Sahlqvist formulae (see e.g. [30]). Similarly, prefixed tableaux calculi and cut-free display calculi can be defined for NIL using the general results from [19, 2]. Moreover, a linear-time transformation from NIL satisfiability problem into the fragment of first-order logic with three individual variables can be given by using the standard translation from modal logics into classical logic (see e.g. [3]). Although this fragment of classical logic is known to be undecidable, the results in the paper can be viewed as an explanation for the decidability (and indeed PSPACE-completeness) of the exact fragment of first-order logic with three individual variables delineated by the translation from NIL. However, the complexity of NIL satisfiability is really a problematic issue since NIL contains interdependent modal connectives. A complexity lower bound can be easily established since NIL can be viewed as an extension of S4.

Theorem 2.1. NIL satisfiability is logarithmic space hard in PSPACE.

Proof:

Let $X$ be the set of NIL satisfiable formulae $\phi$ such that neither $[\sigma]$ nor $[\geq]$ occurs in $\phi$. We shall show that $X$ is the set of S4 satisfiable formulae modulo the replacement of $[\leq]$ by the standard modal connective []. Since S4 satisfiability is logarithmic space hard in PSPACE [20], so is NIL satisfiability. If $\phi \in X$, then $\phi$ is S4 satisfiable since by removing from a NIL-model for $\phi$ the components $R_{\sigma}$ and $R_{\geq}$we get an S4-model. Moreover, if $\phi$ is S4 satisfiable, then there exist an S4-model $\mathcal{M}=\langle W, R, m\rangle$ and $w \in W$ verifying $\mathcal{M}, w \models \phi$. Let $\mathcal{M}^{\prime}=\left\langle W, R_{\leq}, R_{\geq}, R_{\sigma}, m\right\rangle$ be the NIL-model such that $R_{\leq} \stackrel{\text { def }}{=} R, R_{\geq} \stackrel{\text { def }}{=} R^{-1}$ (the converse of $R$ ) and $R_{\sigma} \stackrel{\text { def }}{=} W \times W$. Obviously $\mathcal{M}^{\prime}, w \models \phi$ (modulo the replacement of $[\leq]$ by []) and $\mathcal{M}^{\prime}$ is indeed a NIL-model.

What is done in the proof of Theorem 2.1 at the level of Kripke-style structures can be also interpreted at the level of information systems. Indeed, let $S=\langle O B, A T\rangle$ and $S^{\prime}=\left\langle O B, A T^{\prime}\right\rangle$ be information systems such that

- $A T^{\prime} \stackrel{\text { def }}{=}\left\{a^{\prime}: a \in A T\right\}$; 
- for $a^{\prime} \in A T^{\prime}, V a l_{a^{\prime}} \stackrel{\text { def }}{=} V a l_{a} \cup\{D u m m y\}$ with Dummy $\notin \bigcup\left\{V a l_{a}: a \in A T\right\}$;

- for $a^{\prime} \in A T^{\prime}$ and $x \in O B, a^{\prime}(x) \stackrel{\text { def }}{=} a(x) \cup\{D u m m y\}$.

One can easily check that $\operatorname{fin}(A T)=\operatorname{fin}\left(A T^{\prime}\right)$ and $\operatorname{sim}\left(A T^{\prime}\right)=O B \times O B$, that is the S4-frame $\langle O B, \operatorname{fin}(A T)\rangle$ can be extended to the NIL-frame

$$
\left\langle O B, \operatorname{fin}\left(A T^{\prime}\right), \operatorname{bin}\left(A T^{\prime}\right), \operatorname{sim}\left(A T^{\prime}\right)\right\rangle
$$

where $\operatorname{sim}\left(A T^{\prime}\right)$ is the universal relation $O B \times O B$.

Results from $[20,31,6,21]$ easily entail the following:

Theorem 2.2. (I) Any fragment of NIL with a single modal connective $[\mathrm{R}]$ where $\mathrm{R} \in\{\leq, \geq$ $, \sigma\}$ has a PSPACE-complete satisfiability problem;

(II) The fragment of NIL without the modal connective $[\sigma]$ has a PSPACE-complete satisfiability problem.

It remains to show that NIL satisfiability with the three modal connectives $[\leq],[\geq]$ and $[\sigma]$ living together and interacting, is also in PSPACE. To do so, we shall follow the proof technique from [31] and we propose substantial modifications in order to cope with the much more difficult NIL case. It is worth mentioning that interactions between modal connectives can lead to an increase of computational complexity. K-satisfiability and S5-satisfiability are in PSPACE and in NP, respectively [20]. However, the bimodal logic with an S5 modal connective [1] and with a $\mathrm{K}$ modal connective [2] such that $[1] \phi \Rightarrow[2] \phi$ is an additional axiom has an EXPTIME-hard satisfiability problem [17]. This is well-known that the axiom schema [1] $\phi \Rightarrow[2] \phi$ corresponds to the semantical condition $R_{2} \subseteq R_{1}$ where $R_{i}$ is the accessibility relation associated to the modal operator $[i](i=1,2)$.

\section{Preliminary results}

In Definition 3.1 below, we introduce a closure operator for sets of NIL-formulae as it is done for Propositional Dynamic Logic PDL by Fischer and Ladner in [12].

Definition 3.1. Let $X$ be a set of NIL-formulae. Let $\operatorname{cl}(X)$ be the smallest set of formulae such that:

- $X \subseteq \operatorname{cl}(X)$;

- if $\neg \phi \in \operatorname{cl}(X)$, then $\phi \in \operatorname{cl}(X)$;

- if $\phi_{1} \wedge \phi_{2} \in \operatorname{cl}(X)$, then $\phi_{1}, \phi_{2} \in \operatorname{cl}(X)$;

- if $[\leq] \phi \in \operatorname{cl}(X)$, then $\phi \in \operatorname{cl}(X)$;

- if $[\geq] \phi \in \operatorname{cl}(X)$, then $\phi \in \operatorname{cl}(X)$;

- if $[\sigma] \phi \in \operatorname{cl}(X)$, then $[\geq] \phi \in \operatorname{cl}(X)$; 
- if $[\sigma] \phi \in \operatorname{cl}(X)$ and $\phi$ is not a $[\leq]$-formula, then $[\sigma][\leq] \phi \in \operatorname{cl}(X)$;

- if $[\sigma][\leq] \phi \in \operatorname{cl}(X)$, then $[\sigma] \phi \in \operatorname{cl}(X)$.

Consequently, if $[\sigma] \phi \in \operatorname{cl}(X)$ and $\phi$ is not a $[\leq]$-formula, then $[\leq] \phi \in \operatorname{cl}(X)$ and if $[\sigma] \phi \in$ $\operatorname{cl}(X)$, then $\phi \in \operatorname{cl}(X)$. A set $X$ of formulae is said to be closed $\stackrel{\text { def }}{\Leftrightarrow} \operatorname{cl}(X)=X$. For any finite set $X$ of formulae, we have $\operatorname{md}(\operatorname{cl}(X)) \leq \operatorname{md}(X)+1$. Moreover, for any formula $\psi$, $\operatorname{md}([\leq] \psi)=\operatorname{md}([\geq] \psi)=\operatorname{md}(\operatorname{cl}(\{[\leq] \psi\}))=\operatorname{md}(\operatorname{cl}(\{[\geq] \psi\}))$.

Lemma 3.1. Let $\phi$ be a formula. Then, $\operatorname{card}(\operatorname{cl}(\{\phi\}))<5 \times|\phi|$.

\section{Proof:}

Let $\operatorname{sub}(\phi)$ be the set of subformulae of the formula $\phi$. Obviously, $\operatorname{sub}(\phi) \subseteq \operatorname{cl}(\{\phi\})$. Moreover, $\operatorname{cl}(\{\phi\})$ is the union of the following sets:

1. $\operatorname{sub}(\phi)$

2. $\{[\geq] \psi:[\sigma] \psi \in \operatorname{sub}(\phi)\}$;

3. $\left\{[\geq] \psi:[\sigma][\leq]^{n} \psi \in \operatorname{sub}(\phi), n \geq 1\right\}$;

4. $\left\{[\geq][\leq] \psi:[\sigma] \psi \in \operatorname{sub}(\phi), \psi \neq[\leq] \psi^{\prime}\right\}$;

5. $\left\{[\sigma][\leq] \psi:[\sigma] \psi \in \operatorname{sub}(\phi), \psi \neq[\leq] \psi^{\prime}\right\}$;

6. $\left\{[\sigma] \psi:[\sigma][\leq]^{n} \psi \in \operatorname{sub}(\phi), n \geq 1\right\}$;

7. $\left\{[\leq] \psi:[\sigma] \psi \in \operatorname{sub}(\phi), \psi \neq[\leq] \psi^{\prime}\right\}$.

Each set above is of the cardinality at most $\operatorname{card}(\operatorname{sub}(\phi))$ and a formula in $\operatorname{sub}(\phi)$ can generate at most four formulae in $\operatorname{cl}(\{\phi\})$. So $\operatorname{card}(\operatorname{cl}(\{\phi\}))<5 \times|\phi|$, since $\operatorname{card}(\operatorname{sub}(\phi))<|\phi|$.

In order to determine the NIL satisfiability of some formula $\phi$, we need to handle sets of formulae. All those sets shall be subsets of $\operatorname{cl}(\{\phi\})$. In establishing the PSPACE complexity upper bound, the fact that $\operatorname{cl}(\{\phi\})$ is finite and its cardinality is linear in the size of $\phi$ plays an important role.

In order to check whether $\phi$ is NIL-satisfiable, we build sequences of the form

$$
X_{0} x_{0} X_{1} x_{1} X_{2} x_{2} \ldots
$$

where $\phi \in X_{0} \subseteq \operatorname{cl}(\{\phi\})$ and for $i \in \omega, X_{i}$ is a consistent subset of $\operatorname{cl}(\{\phi\})$ and $x_{i} \in\{\sigma, \leq, \geq\}$. We extend a finite sequence $X_{0} x_{0} X_{1} x_{1} \ldots x_{i-1} X_{i}$ with $x_{i} X_{i+1}$ whenever we need a witness of $\left[x_{i}\right] \psi \notin X_{i}$ for some formula $\psi$ (and $\psi \notin X_{i+1}$ ). The intention is to build paths in some NIL model $\mathcal{M}=\left\langle W, R_{\leq}, R_{\geq}, R_{\sigma}, m\right\rangle$ such that for $i \in \omega$, there is $w_{i} \in W$ such that $\mathcal{M}, w_{i} \models \psi$ iff $\psi \in X_{i}$ and $\left\langle w_{i}, w_{i+1}\right\rangle \in R_{x_{i}}$. This roughly corresponds to the exploration of a branch in the depth-first proof search for a tableaux-style calculus. In order to avoid confusion, $\sigma$ [resp. $\leq$, $\geq$ ] shall be written sim [resp. fin, bin]. 
In order to establish termination of the proof of building sequences, which is a necessary step to obtain the PSPACE complexity upper bound, we define subsets $\operatorname{cl}(s, \phi) \subseteq \operatorname{cl}(\{\phi\})$ for $s \in\{\operatorname{sim}, \text { fin, } \operatorname{bin}\}^{*}$ such that for $i \in \omega$,

$$
X_{i} \subseteq \operatorname{cl}\left(x_{0} \ldots x_{i-1}, \phi\right)
$$

For $s \in\{\operatorname{sim}, \text { fin, } \operatorname{bin}\}^{*}$ and $x \in\{\operatorname{sim}$, fin, $\operatorname{bin}\} \mathrm{cl}(s \cdot x, \phi)$ contains all the formulae $\psi$ which we could possibly be put in $X_{i+1}$ for $\psi \in \operatorname{cl}\left(x_{0} \ldots x_{i-1}, \phi\right)$.

We will get termination if there is some computable map $f: \omega \rightarrow \omega$ such that for $|s| \geq f(|\phi|)$, $\operatorname{cl}(s, \phi)=\emptyset$. To establish the PSPACE complexity upper bound, $f$ should preferably be bounded by a polynomial. Those general principles may look quite attractive but in concrete examples of modal logics they are seldom sufficient to show that the satisfiability problem is in PSPACE. Since transitivity of $R_{\leq}$is required, if $[\leq] \psi \in X_{i}$, then $\mathcal{M}, w_{i}=[\leq] \psi, \mathcal{M}, w_{i}=[\leq$ ]$[\leq] \psi$ and therefore one can expect that $[\leq] \psi \in X_{i+1}$ if $x_{i}=$ fin. So the formula $[\leq] \psi \in X_{i}$ should be propagated for any "fin" transition. However, this does not guarantee termination. Actually, as already known from [20,31,5], duplicates can be identified in $X_{0} x_{0} X_{1} x_{1} X_{2} x_{2} \ldots$ which corresponds to a cycle detection (see also [13]). Since $\operatorname{card}(\mathcal{P}(\operatorname{cl}(\{\phi\})))$ is in $\mathcal{O}\left(2^{|\phi|}\right)$, a finer analysis is necessary to establish the PSPACE complexity upper bound as done in [20] (see also [31] for the tense extension of Ladner's solution). Things are even worse. since by (N4), if $[\sigma] \psi \in X_{i}$, then $\mathcal{M}, w_{i}=[\sigma] \psi$ and $\mathcal{M}, w_{i}=[\geq]^{n}[\sigma][\leq]^{n^{\prime}} \psi$ for $n, n^{\prime} \geq 0$. One can expect that $[\geq]^{n-1}[\sigma][\leq]^{n^{\prime}} \psi \in X_{i+1}$ if $x_{i}=$ bin and $n \geq 1$. This is not a good perspective for termination and in this paper we shall also provide a technical answer to this problem. We omit at this stage of the paper, the complications caused by having a symmetric relation in the NIL models.

In order to conclude this introductory part that motivates the existence of the sets of the form $\operatorname{cl}(s, \phi)$, let us say that once the set $X_{i}$ of formulae is built and $x_{i}$ is chosen, the set $X_{i+1}$ satisfies:

1. $X_{i+1}$ is a consistent subset of $\operatorname{cl}\left(x_{0} \ldots x_{i}, \phi\right)$;

2. $\left\langle X_{i}, X_{i+1}\right\rangle$ satisfies a certain condition (to be specified in the sequel) that shall guarantee that $\mathcal{M}$ is a NIL model and $\left\langle w_{i}, w_{i+1}\right\rangle \in R_{x_{i}}$.

Definition 3.2. Let $\phi$ be a formula. For $s \in\{\operatorname{sim}, \operatorname{fin}, \operatorname{bin}\}^{*}$, let $\operatorname{cl}(s, \phi)$ be the smallest set such that:

1. $\operatorname{cl}(\lambda, \phi)=\operatorname{cl}(\{\phi\})$

2. $\operatorname{cl}(s, \phi)$ is closed;

3. if $[\sigma][\leq] \psi \in \operatorname{cl}(s, \phi)$, then $[\leq] \psi \in \operatorname{cl}(s \cdot \operatorname{sim}, \phi)$;

4. if $[\leq] \psi \in \operatorname{cl}(s, \phi)$, then $[\leq] \psi \in \operatorname{cl}(s \cdot$ fin, $\phi)$;

5. if $[\geq] \psi \in \operatorname{cl}(s, \phi)$, then $[\geq] \psi \in \operatorname{cl}(s \cdot \operatorname{bin}, \phi)$;

6. if $[\sigma][\leq] \psi \in \operatorname{cl}(s, \phi)$, then $[\sigma][\leq] \psi \in \operatorname{cl}(s \cdot \operatorname{bin}, \phi)$. 
Example 3.1. Let $\phi$ be the formula $[\sigma] \mathrm{p}_{0}$. We have

$$
\operatorname{cl}(\lambda, \phi)=\operatorname{cl}(\{\phi\})=\left\{[\sigma] \mathrm{p}_{0}, \mathrm{p}_{0},[\geq] \mathrm{p}_{0},[\sigma][\leq] \mathrm{p}_{0},[\geq][\leq] \mathrm{p}_{0},[\leq] \mathrm{p}_{0}\right\}
$$

Below are some examples of sets of the form $\operatorname{cl}(s, \phi)$ :

- $\operatorname{cl}($ bin, $\phi)=\operatorname{cl}(\{\phi\})$;

- $\operatorname{cl}($ bin $\cdot \operatorname{sim}, \phi)=\left\{\mathrm{p}_{0},[\leq] \mathrm{p}_{0}\right\}$;

- $\operatorname{cl}($ bin $\cdot \operatorname{sim} \cdot$ fin, $\phi)=\left\{\mathrm{p}_{0},[\leq] \mathrm{p}_{0}\right\}$;

- $\operatorname{cl}($ bin $\cdot \operatorname{sim} \cdot$ fin $\cdot \operatorname{bin}, \phi)=\emptyset$.

One can check that for any $s \in\{\operatorname{sim}, \operatorname{bin}, \operatorname{fin}\}^{*}$ such that $|s| \geq 4$, we have $\operatorname{cl}(s, \phi)=\emptyset$.

Lemma 3.2 contains some basic properties of the sets $\operatorname{cl}(s, \phi)$.

Lemma 3.2. Let $\phi$ be a formula and $s, s^{\prime} \in\{\operatorname{sim}, \text { fin, bin }\}^{*}$ such that $s$ is a prefix of $s^{\prime}$. Then,

(I) $\operatorname{cl}\left(s^{\prime}, \phi\right) \subseteq \operatorname{cl}(s, \phi)$;

(II) if $[\sigma] \psi \in \operatorname{cl}(s, \phi)$, then $\psi \in \operatorname{cl}(s \cdot \operatorname{sim}, \phi)$;

(III) if $[\sigma] \psi \in \operatorname{cl}(s, \phi)$, then $[\sigma] \psi \in \operatorname{cl}(s \cdot \operatorname{bin}, \phi)$;

(IV) if $[\leq] \psi \in \operatorname{cl}(s \cdot$ bin, $\phi)$, then $\operatorname{md}([\leq] \psi)<\operatorname{md}(\operatorname{cl}(s \cdot$ bin, $\phi))$.

\section{Proof:}

(I) This is immediate by Definition 3.2, since both $\operatorname{cl}(s, \phi)$ and $\operatorname{cl}\left(s^{\prime}, \phi\right)$ are closed.

(II) Let $[\sigma] \psi \in \operatorname{cl}(s, \phi)$. If $\psi=[\leq] \psi^{\prime}$, then by definition $\psi \in \operatorname{cl}(s \cdot \operatorname{sim}, \phi)$. If $\psi \neq[\leq] \psi^{\prime}$, then since $\operatorname{cl}(s, \phi)$ is closed, $[\sigma][\leq] \psi \in \operatorname{cl}(s, \phi)$. By definition, $[\leq] \psi \in \operatorname{cl}(s \cdot \operatorname{sim}, \phi)$. Since $\operatorname{cl}(s \cdot \operatorname{sim}, \phi)$ is closed, $\psi \in \operatorname{cl}(s \cdot \operatorname{sim}, \phi)$.

(III) Similar to (II).

(IV) Let $[\leq] \psi \in \operatorname{cl}(s \cdot \operatorname{bin}, \phi)$. Two cases are distinguished according to the way the formula $[\leq] \psi$ can appear in $\operatorname{cl}(s \cdot$ bin, $\phi)$.

Case 1: $[\leq] \psi \in \operatorname{cl}\left(\left\{[\geq] \psi^{\prime}\right\}\right)$ for some $[\geq] \psi^{\prime} \in \operatorname{cl}(s, \phi)$ (see Definition 3.2(5)).

We distinguish two cases according to the way $[\leq] \psi$ belongs to $\operatorname{cl}\left(\left\{[\geq] \psi^{\prime}\right\}\right)$. If $[\leq] \psi$ is a subformula of $[\geq] \psi^{\prime}$ (see the point 1. in the proof of Lemma 3.1), then $\operatorname{md}([\leq] \psi)<\operatorname{md}\left([\geq] \psi^{\prime}\right)$ since $[\leq] \psi \neq[\geq] \psi^{\prime}$ and $[\geq] \psi^{\prime} \in \operatorname{cl}(s \cdot \operatorname{bin}, \phi)$. So $\operatorname{md}([\leq] \psi)<\operatorname{md}(\operatorname{cl}(s \cdot \operatorname{bin}, \phi))$. If $[\sigma] \psi$ is a subformula of $[\geq] \psi^{\prime}$ and $\psi$ is not a $[\leq]$-formula (see the point 7 . in the proof of Lemma 3.1), then $[\sigma][\leq] \psi \in \operatorname{cl}\left(\left\{[\geq] \psi^{\prime}\right\}\right) \subseteq \operatorname{cl}(s \cdot$ bin, $\phi)$. Hence, $\operatorname{md}([\leq] \psi)<\operatorname{md}(\operatorname{cl}(s \cdot$ bin, $\phi))$.

Case 2: $[\leq] \psi \in \operatorname{cl}\left(\left\{[\sigma][\leq] \psi^{\prime}\right\}\right)$ for some $[\sigma][\leq] \psi^{\prime} \in \operatorname{cl}(s, \phi)$ (see Definition 3.2(6)).

Thus,

- $[\leq] \psi \in \operatorname{cl}\left(\left\{[\leq] \psi^{\prime}\right\}\right)$; and

- $\operatorname{md}([\leq] \psi) \leq \operatorname{md}\left([\leq] \psi^{\prime}\right)=\operatorname{md}\left(\operatorname{cl}\left(\left\{[\leq] \psi^{\prime}\right\}\right)\right)$.

Hence, $\operatorname{md}([\leq] \psi) \leq \operatorname{md}\left([\leq] \psi^{\prime}\right)<\operatorname{md}\left([\sigma][\leq] \psi^{\prime}\right) \leq \operatorname{md}(\operatorname{cl}(s \cdot \operatorname{bin}, \phi))$. 
Lemma 3.3 below is crucial to prove forthcoming Theorem 3.1 and this is the cornerstone for termination of the procedure defined in Section 4.1. It states sufficient conditions on the string $s^{\prime}$ to guarantee that the modal degree of $\operatorname{cl}\left(s \cdot s^{\prime}, \phi\right)$ is strictly less than the modal degree of $\operatorname{cl}(s, \phi)$.

Lemma 3.3. Let $\phi$ be a formula, $s \in\{\text { sim, fin,bin }\}^{*}$ and $s^{\prime} \in\{$ bin.fin.bin, fin.bin.fin, sim $\}$. Then, $\operatorname{md}\left(\operatorname{cl}\left(s \cdot s^{\prime}, \phi\right)\right) \leq \max (0, \operatorname{md}(\operatorname{cl}(s, \phi))-1)$.

\section{Proof:}

Assume that $\operatorname{md}(\operatorname{cl}(s, \phi)) \geq 1$, otherwise the proof is immediate by Lemma 3.2(I). Let $\varphi \in \operatorname{cl}(s, \phi)$ be such that $\operatorname{md}(\varphi)=\operatorname{md}(\operatorname{cl}(s, \phi))$. Let us show that $\varphi \notin \operatorname{md}\left(\operatorname{cl}\left(s \cdot s^{\prime}, \phi\right)\right)$. By Lemma 3.2(I) we are done. We distinguish cases according to the value of $s^{\prime}$.

Case 1: $s^{\prime}=$ fin $\cdot$ bin $\cdot$ fin

Case 1.1: the outermost connective of $\varphi$ is in $\{\wedge, \neg\}$.

By Definition 3.2, we have $\varphi \in \operatorname{cl}(s \cdot f i n, \phi)$ iff there is $[\leq] \psi \in \operatorname{cl}(s, \phi)$ such that $\varphi \in \operatorname{cl}(\{[\leq] \psi\})$. Suppose $\varphi \in \operatorname{cl}($ s $\cdot$ fin,$\phi) \cdot \varphi$ can only be a proper subformula of $[\leq] \psi$. But then $\operatorname{md}(\varphi)<\operatorname{md}([\leq$ ]$\psi)$, a contradiction. Hence, $\varphi \notin \operatorname{cl}(s \cdot$ fin,$\phi)$ and therefore $\varphi \notin \operatorname{cl}\left(s \cdot s^{\prime}, \phi\right)$.

Case 1.2: $\varphi=[\sigma][\leq] \varphi^{\prime}$.

Suppose $\varphi \in \operatorname{cl}(s \cdot$ fin,$\phi)$ (otherwise $\varphi \notin \operatorname{cl}\left(s \cdot s^{\prime}, \phi\right)$ by Lemma 3.2(I)). By Definition 3.2(4), there is $[\leq] \psi \in \operatorname{cl}(s, \phi)$ such that $\varphi \in \operatorname{cl}(\{[\leq] \psi\}) .[\sigma][\leq] \varphi^{\prime}$ cannot be a subformula of $[\leq] \psi$, otherwise $\operatorname{md}\left([\sigma][\leq] \varphi^{\prime}\right)<\operatorname{md}([\leq] \psi)$ (see the point 1. in the proof of Lemma 3.1). Similarly, $[\sigma][\leq]^{n+1} \varphi^{\prime}$ is not a subformula of $[\leq] \psi$ with $n \geq 1$ (see the point 3. in the proof of Lemma 3.2) by maximality of $\operatorname{md}(\varphi)$. The only remaining possibility is that $[\sigma] \varphi^{\prime}$ is a subformula of $[\leq] \psi$ and $\varphi^{\prime}$ is not a $[\leq]$-formula. Hence, $\operatorname{md}([\leq] \psi)=\operatorname{md}(\varphi)$. Now since $\varphi \in \operatorname{cl}(s \cdot f i n \cdot \operatorname{bin}, \phi), \operatorname{md}(\varphi)=\operatorname{md}(\operatorname{cl}(s$. fin $\cdot \operatorname{bin}, \phi))$. By Lemma 3.2(IV), for any $[\leq] \psi^{\prime}$ in $\operatorname{cl}(s, \phi)$ such that $\operatorname{md}\left([\leq] \psi^{\prime}\right)=\operatorname{md}(\operatorname{cl}(s, \phi))$, we have $[\leq] \psi^{\prime} \notin \operatorname{cl}(s \cdot$ fin $\cdot$ bin,$\phi)$. In particular, $[\leq] \psi \notin \operatorname{cl}(s \cdot f$ in $\cdot$ bin,$\phi)$. We can now in position to conclude the present case. $[\sigma][\leq] \varphi^{\prime} \in \operatorname{cl}\left(s \cdot s^{\prime}, \phi\right)$ iff there is $[\leq] \psi^{\prime} \in \operatorname{cl}(s \cdot f i n \cdot b i n, \phi)$ such that $[\sigma][\leq] \varphi^{\prime} \in \operatorname{cl}\left(\left\{[\leq] \psi^{\prime}\right\}\right)$ (see Definition 3.2(4)). Suppose $[\sigma][\leq] \varphi^{\prime} \in \operatorname{cl}\left(s \cdot s^{\prime}, \phi\right)$. So $\operatorname{md}\left([\sigma][\leq] \varphi^{\prime}\right)=$ $\operatorname{md}\left([\leq] \psi^{\prime}\right)$ and from the above developments, we can conclude that $[\leq] \psi^{\prime} \notin \operatorname{cl}(s \cdot$ fin $\cdot \operatorname{bin}, \phi)$, a contradiction.

Case 1.3: $\varphi=[\leq] \varphi^{\prime}$.

So $\varphi \in \operatorname{cl}(s \cdot f i n, \phi)$. Now suppose $\varphi \in \operatorname{cl}(s \cdot f i n \cdot$ bin, $\phi)$. By Lemma 3.2(IV $), \operatorname{md}(\varphi)<$ $\operatorname{md}(\mathrm{cl}(s \cdot f$ in $\cdot \operatorname{bin}, \phi))$. By Lemma 3.2(I),

$$
\operatorname{md}(\operatorname{cl}(s \cdot f i n \cdot b i n, \phi)) \leq \operatorname{md}(\operatorname{cl}(s, \phi))
$$

So, $\operatorname{md}(\varphi)<\operatorname{md}(\operatorname{cl}(s, \phi))$, a contradiction.

Case 1.4: $\varphi=[\geq] \varphi^{\prime}$.

Assume $\varphi \in \operatorname{cl}(s \cdot f i n, \phi)$ (otherwise $\varphi \notin \operatorname{cl}\left(s \cdot s^{\prime}, \phi\right)$ by Lemma 3.2(I)). So, there is $[\leq] \psi \in \operatorname{cl}(s, \phi)$ such that $\varphi \in \operatorname{cl}(\{[\leq] \psi\})$. So $\operatorname{md}([\leq] \psi)=\operatorname{md}(\varphi)$. Since $\operatorname{md}(\varphi)=\operatorname{md}(\operatorname{cl}(s, \phi)), \varphi$ cannot be a subformula of $[\leq] \psi$ (see the point 1. in the proof of Lemma 3.1). Similarly, there is no subformula $[\sigma][\leq]^{n} \varphi^{\prime}$ of $[\leq] \psi$ for some $n \geq 1$ (see the point 3. in the proof of Lemma 3.1) by maximality 
of $\operatorname{md}(\varphi) . \varphi$ can only be of the form $[\geq][\leq] \varphi^{\prime \prime}$ for some subformula $[\sigma] \varphi^{\prime \prime}$ of $[\leq] \psi$ such that $\varphi^{\prime \prime}$ is not a $[\leq]$-formula. So $\varphi \in \operatorname{cl}(s \cdot$ fin $\cdot$ bin, $\phi)$. By Lemma $3.2(\mathrm{IV}),[\leq] \psi \notin \operatorname{cl}(s \cdot$ fin $\cdot$ bin, $\phi)$. Otherwise, we have

$$
\operatorname{md}(\varphi)=\operatorname{md}([\leq] \psi)=\operatorname{md}(\operatorname{cl}(s \cdot \text { fin } \cdot \operatorname{bin}, \phi))
$$

Actually, for any formula $[\leq] \psi^{\prime} \in \operatorname{cl}(s, \phi)$ such that $\operatorname{md}\left([\leq] \psi^{\prime}\right)=\operatorname{md}(\operatorname{cl}(s, \phi))$, we have $[\leq] \psi^{\prime} \notin$ $\mathrm{cl}(s \cdot$ fin $\cdot$ bin,$\phi)$.

Now, $[\geq][\leq] \varphi^{\prime \prime} \in \operatorname{cl}(s \cdot$ fin $\cdot$ bin $\cdot$ fin, $\phi)$ iff there is $[\leq] \psi^{\prime} \in \operatorname{cl}(s \cdot$ fin $\cdot$ bin, $\phi)$ such that $[\geq][\leq] \varphi^{\prime \prime} \in \operatorname{cl}\left(\left\{[\leq] \psi^{\prime}\right\}\right.$ ) (see Definition 3.2(4)). Analogously, we can conclude that $[\sigma] \varphi^{\prime \prime}$ is a subformula of $[\leq] \psi^{\prime}$. Suppose $[\geq][\leq] \varphi^{\prime \prime} \in \operatorname{cl}(s \cdot$ fin $\cdot$ bin $\cdot$ fin,$\phi)$. By Lemma 3.2(I), we have $\operatorname{cl}(s \cdot$ fin $\cdot$ bin,$\phi) \subseteq \operatorname{cl}(s, \phi)$ and therefore $[\leq] \psi^{\prime} \in \operatorname{cl}(s, \phi)$ and $\operatorname{md}\left([\leq] \psi^{\prime}\right)=\operatorname{md}(\varphi)$ which leads to a contradiction from the above developments. Thus, $[\geq][\leq] \varphi^{\prime \prime} \notin \operatorname{cl}(s \cdot$ fin $\cdot$ bin $\cdot$ fin,$\phi)$.

Case 2: $s^{\prime}=$ bin $\cdot$ fin $\cdot$ bin

Case 2.1: the outermost connective of $\varphi$ is in $\{\wedge, \neg\}$.

See the Case 1.1.

Case 2.2: either $\varphi=[\sigma][\leq] \varphi^{\prime}$ or $\varphi=[\geq] \varphi^{\prime}$ (see Definition 3.2(5-6)).

So $\varphi \in \operatorname{cl}(s \cdot \operatorname{bin}, \phi)$. Suppose $\varphi \in \operatorname{cl}\left(s \cdot \operatorname{bin} \cdot\right.$ fin, $\phi$ ) (otherwise $\varphi \notin \operatorname{cl}\left(s \cdot s^{\prime}, \phi\right)$ ). So there is $[\leq] \psi \in \operatorname{cl}(s \cdot \operatorname{bin}, \phi)$ such that $\varphi \in \operatorname{cl}(\{[\leq] \psi\})$ (see Definition 3.2(4)). Since $\operatorname{md}(\varphi)=\operatorname{md}(\operatorname{cl}(s, \phi))$, $\operatorname{md}([\leq] \psi)=\operatorname{md}(\varphi)$. By Lemma 3.2(IV), $\operatorname{md}(\varphi)<\operatorname{md}(\operatorname{cl}(s \cdot b i n, \phi))$, a contradiction.

Case 2.3: $\varphi=[\leq] \varphi^{\prime}$.

Suppose $\varphi \in \operatorname{cl}(s \cdot \operatorname{bin}, \phi)$. By Lemma 3.2(IV), $\operatorname{md}(\varphi)<\operatorname{md}(\operatorname{cl}(s \cdot \operatorname{bin}, \phi)) \leq \operatorname{md}(\operatorname{cl}(s, \phi))$, a contradiction.

Case 3: $s^{\prime}=$ sim

Suppose $\varphi \in \operatorname{cl}(s \cdot \operatorname{sim}, \phi)$. By Definition 3.2(3), $\varphi \in \operatorname{cl}(s \cdot \operatorname{sim}, \phi)$ iff there is $[\sigma][\leq] \psi \in \operatorname{cl}(s, \phi)$ such that $\varphi \in \operatorname{cl}(\{[\leq] \psi\})$. Observe that $\operatorname{md}([\leq] \psi)=\operatorname{md}(\operatorname{cl}(\{[\leq] \psi\}))<\operatorname{md}([\sigma][\leq] \psi)$. Hence $\operatorname{md}(\varphi)<\operatorname{md}(\operatorname{cl}(\{[\leq] \psi\}))$, a contradiction.

Theorem 3.1. Let $\phi$ be a formula and $s \in\{\operatorname{sim}, \text { fin, bin }\}^{*}$ be such that neither bin $\cdot$ bin nor fin. fin is a substring of $s$ and $|s| \geq 3 \times|\phi|$. Then $\operatorname{cl}(s, \phi)=\emptyset$.

\section{Proof:}

First observe that for any substring $s^{\prime}$ of length 3 in $s$, either sim occurs in $s^{\prime}$ or $s^{\prime} \in\{$ fin bin. fin, bin $\cdot$ fin $\cdot$ bin $\}$. Since $\operatorname{md}(\phi)+1 \leq|\phi|$, let $s^{\prime \prime}$ be the prefix of $s$ of length $3 \times \operatorname{md}(\phi)$. By Lemma $3.3, \operatorname{md}\left(\operatorname{cl}\left(s^{\prime \prime}, \phi\right)\right)=0$ and therefore $\operatorname{cl}\left(s^{\prime}, \phi\right)=\emptyset$.

What is really important in Theorem 3.1 is that for certain elements $s$ of $\{\operatorname{sim}, \text { fin, bin }\}^{*}$ of polynomial length in $|\phi|, \operatorname{cl}(s, \phi)$ is empty. The strings bin · bin and fin f fin do not occur in $s$, since by (N1) and (N2) we shall identify any element of $\{\text { bin }\}^{+}\left[\right.$resp. $\left\{\text {fin }^{+}\right\}^{+}$with bin [resp. fin]. Theorem 3.1 can be extended in the following way.

Theorem 3.2. Let $f: \omega \rightarrow \omega$ be a computable map, $\phi$ be a formula and $s \in\{\operatorname{sim} \text {, fin, bin }\}^{*}$ be such that neither bin ${ }^{k+1}$ nor fin ${ }^{k+1}$ is a substring of $s$ for $k \geq f(|\phi|)$ and $|s| \geq 3 \times|\phi| \times f(|\phi|)$. Then $\operatorname{cl}(s, \phi)=\emptyset$. 
Theorem 3.1 is a particular case of Theorem 3.2 where $f$ is the constant map 1 . The proof of Theorem 3.2 also uses the property that $\operatorname{cl}\left(s \cdot b i n^{k}, \phi\right)=\operatorname{cl}(s \cdot b i n, \phi)\left[\operatorname{resp} \cdot \operatorname{cl}\left(s \cdot f i n^{k}, \phi\right)=\right.$ $\mathrm{cl}(s \cdot f$ in,$\phi)]$ for $k \geq 1$.

It is legitimate to wonder whether Theorem 3.1 is optimal. We show that we can hardly do better: the factor 3 cannot be replaced by the factor 2 .

Lemma 3.4. For $n \in \omega$, there exist a formula $\phi_{n}$ and $s_{n} \in\{\text { sim, bin, fin }\}^{*}$ such that

- $\operatorname{md}\left(\phi_{n}\right)=n+1$ and $\left|\phi_{n}\right|=n+3$;

- neither bin bin nor fin fin occurs in $s_{n}$;

- $\left|s_{n}\right|=3 \times n+1$;

- $\operatorname{cl}\left(s_{n}, \phi_{n}\right) \neq \emptyset$.

\section{Proof:}

Let us define $\phi_{n} \stackrel{\text { def }}{=}[\leq][\sigma]^{n} \mathrm{p}_{0}$ and $s_{n} \stackrel{\text { def }}{=}(\text { fin } \cdot \operatorname{bin} \cdot \operatorname{sim})^{n} \cdot$ fin. Let us show that $\mathrm{cl}\left(s_{n}, \phi_{n}\right) \neq \emptyset$. To do so, we prove that for $i \in\{0, \ldots, n\},[\leq][\sigma]^{i} \mathrm{p}_{0} \in \operatorname{cl}\left((\text { fin } \cdot \text { bin } \cdot \operatorname{sim})^{n-i}, \phi_{n}\right)$.

Base case: $i=n .[\leq][\sigma]^{n} \mathrm{p}_{0} \in \operatorname{cl}\left(\lambda, \phi_{n}\right)$.

Induction step: Let us take as an induction hypothesis that $[\leq][\sigma]^{i+1} \mathrm{p}_{0} \in \operatorname{cl}\left((\text { fin } \cdot \text { bin } \cdot \text { sim })^{n-i-1}, \phi_{n}\right)$ for some $i \geq 0$. It follows that

- $[\leq][\sigma]^{i+1} \mathrm{p}_{0} \in \operatorname{cl}\left((\text { fin } \cdot \text { bin } \cdot \text { sim })^{n-i-1} \cdot\right.$ fin,$\left.\phi_{n}\right)$;

- $[\sigma]^{i+1} \mathrm{p}_{0} \in \operatorname{cl}\left((\text { fin } \cdot \text { bin } \cdot \operatorname{sim})^{n-i-1} \cdot\right.$ fin, $\left.\phi_{n}\right)$;

- $[\sigma][\leq][\sigma]^{i} \mathrm{p}_{0} \in \operatorname{cl}\left((\text { fin } \cdot \text { bin } \cdot \operatorname{sim})^{n-i-1} \cdot\right.$ fin,$\left.\phi_{n}\right)$;

- $[\sigma][\leq][\sigma]^{i} \mathrm{p}_{0} \in \operatorname{cl}\left((\text { fin } \cdot \text { bin } \cdot \operatorname{sim})^{n-i-1} \cdot\right.$ fin $\cdot$ bin,$\left.\phi_{n}\right)$;

- $[\leq][\sigma]^{i} \mathrm{p}_{0} \in \operatorname{cl}\left((\text { fin } \cdot \text { bin } \cdot \operatorname{sim})^{n-i-1} \cdot\right.$ fin $\cdot$ bin $\left.\cdot \operatorname{sim}, \phi_{n}\right)$.

Hence, $[\leq] \mathrm{p}_{0} \in \operatorname{cl}\left((\text { fin } \cdot \text { bin } \cdot \operatorname{sim})^{n}, \phi_{n}\right)$ and $[\leq] \mathrm{p}_{0} \in \operatorname{cl}\left((\text { fin } \cdot \text { bin } \cdot \operatorname{sim})^{n} \cdot\right.$ fin,$\left.\phi_{n}\right)$.

Definition 3.3. The binary relation $\approx$ on sets of NIL-formulae is defined as follows: $X \approx Y$ $\stackrel{\text { def }}{\Leftrightarrow}$

1. for all $[\sigma] \psi \in X, \psi \in Y$;

2. for all $[\sigma] \psi \in Y, \psi \in X$.

The binary relation $\preceq$ is defined as follows: $X \preceq Y \stackrel{\text { def }}{\Leftrightarrow}$

1. for all $[\leq] \psi \in X,[\leq] \psi, \psi \in Y$;

2. for all $[\geq] \psi \in Y,[\geq] \psi, \psi \in X$;

3. for all $[\sigma] \psi \in Y,[\sigma] \psi \in X$.

Let clos be the set of subsets $Y$ of $\operatorname{cl}(\{\phi\})$ such that for $\mathrm{R} \in\{\sigma, \geq, \leq\},[\mathrm{R}] \psi \in Y$ implies $\psi \in Y$. The binary relation $\approx$ is a reflexive and symmetric relation on clos and $\preceq$ is a reflexive and transitive relation on clos. In Definition 3.4 below, for $s \in\{\operatorname{sim}, f i n, b i n\}^{*}$, we define the set of $s$-consistent sets which is a subset of clos. 
Definition 3.4. Let $X$ be a subset of $\operatorname{cl}(s, \phi)$ for some $s \in\{\operatorname{sim}, \text { bin, fin }\}^{*}$ and for some formula $\phi$. The set $X$ is said to be $s$-consistent $\stackrel{\text { def }}{\Leftrightarrow}$ for $\psi \in \operatorname{cl}(s, \phi)$ :

1. if $\psi=\neg \varphi$, then $\varphi \in X$ iff not $\psi \in X$;

2. if $\psi=\varphi_{1} \wedge \varphi_{2}$, then $\left\{\varphi_{1}, \varphi_{2}\right\} \subseteq X$ iff $\psi \in X$;

3. if $\psi=[\mathrm{R}] \varphi$ for some $\mathrm{R} \in\{\sigma, \leq, \geq\}$ and $\psi \in X$, then $\varphi \in X$;

4. if $\psi=[\sigma] \varphi, \varphi \neq[\leq] \varphi^{\prime}$ and $\psi \in X$, then $[\sigma][\leq] \varphi \in X$;

5. if $\psi=[\sigma][\leq] \varphi$ and $\psi \in X$, then $[\sigma] \varphi \in X$;

6. if $\psi=[\sigma] \varphi$ and $\psi \in X$, then $[\geq] \varphi \in X$.

Roughly speaking, the $s$-consistency entails the maximal propositional consistency with respect to the set $\operatorname{cl}(s, \phi)$ of formulae. Furthermore, the modal conditions 3.-6. in Definition 3.4 are added in order to take into account the reflexivity of the relations in the NIL models and the inclusion $R_{\leq} \cup R_{\geq} \subseteq R_{\sigma}$. For instance, if $X$ is $s$-consistent and $[\sigma] \psi \in X$, then $\{[\leq] \psi,[\geq] \psi\} \subseteq X$.

Lemma 3.5. Let $\mathcal{M}=\left\langle W, R_{\leq}, R_{\geq}, R_{\sigma}, m\right\rangle$ be a NIL model, $w \in W, s \in\{\operatorname{sim} \text {, fin,bin }\}^{*}, \phi$ be a NIL formula. Then, $\{\psi \in \operatorname{cl}(s, \phi): \mathcal{M}, w \models \psi\}$ is s-consistent.

The proof of the above lemma is by an easy verification using that $\operatorname{cl}(s, \phi)$ is closed. Lemma 3.6(II) below roughly states that $\preceq \subseteq \approx$ which is the syntactic version of $R_{\leq} \subseteq R_{\sigma}$ in the NIL models. Similarly, Lemma 3.6(I) below states a syntactic version of $R_{\geq} \circ R_{\sigma} \circ R_{\leq} \subseteq R_{\sigma}$ in the NIL models. $\circ$ denote the composition operation for binary relations.

Lemma 3.6. Let $X_{i}$ be an $s_{i}$-consistent set, $i=1, \ldots, 4$, such that $X_{1} \succeq X_{2} \approx X_{3} \preceq X_{4}$. Then,

(I) if $[\sigma] \psi \in X_{1}, \psi \in X_{4}$.

(II) $X_{1} \approx X_{2}$.

\section{Proof:}

(I) Let $[\sigma] \psi \in X_{1}$. Since $X_{1} \succeq X_{2}$, we have $[\sigma] \psi \in X_{2}$ and $\psi \in X_{2}$ by Definition 3.4(3). If $\psi=[\leq] \varphi$, then by $X_{2} \approx X_{3},[\leq] \varphi \in X_{3}$ and by $X_{3} \preceq X_{4},[\leq] \varphi \in X_{4}$. Otherwise, if $\psi \neq[\leq] \varphi$, then by $s_{2}$-consistency, we have $[\sigma][\leq] \psi \in X_{2}$. By $X_{2} \approx X_{3},[\leq] \psi \in X_{3}$. By $X_{3} \preceq X_{4}, \psi \in X_{4}$.

(II) Let $[\sigma] \psi \in X_{2}$. If $\psi \neq[\leq] \varphi$, then $[\sigma][\leq] \psi \in X_{2}$ by $s_{2}$-consistency. Moreover, by $s_{2}$ consistency, $[\leq] \psi \in X_{2}$. Now since $X_{2} \preceq X_{1}, \psi \in X_{1}$. In the case $\psi=[\leq] \varphi$, by $s_{2}$-consistency, $\psi \in X_{2}$. Now since $X_{2} \preceq X_{1},[\leq] \varphi \in X_{1}$.

Let $[\sigma] \psi \in X_{1}$. If $\psi \neq[\leq] \varphi$, then $[\sigma][\leq] \psi \in X_{1}$ by $s_{1}$-consistency. By $s_{1}$-consistency, $[\geq][\leq] \psi \in$ $X_{1}$. Now since $X_{2} \preceq X_{1},[\leq] \psi \in X_{2}$. By $s_{2}$-consistency of $X_{2}, \psi \in X_{2}$. In the case $\psi=[\leq] \varphi$, by $s_{1}$-consistency, $[\geq] \psi \in X_{1}$. Now since $X_{2} \preceq X_{1}, \psi \in X_{2}$.

In the forthcoming Lemma 3.7, Lemma 3.8, Lemma 3.9, Lemma 3.10 and Lemma 3.11 we state some basic facts about the sets $\operatorname{cl}(s, \phi)$.

Lemma 3.7. Let $s \in\{\operatorname{sim}, \text { bin, } f i n\}^{*}$ and $\phi$ be a formula. 
(I) if $[\sigma] \psi \in \operatorname{cl}(s, \phi)$, then $\psi \in \operatorname{cl}(s \cdot \operatorname{sim}, \phi)$;

(II) if $[\sigma] \psi \in \operatorname{cl}(s, \phi)$, then $\psi \in \operatorname{cl}(s \cdot f$ in,$\phi)$;

(III) if $[\sigma] \psi \in \operatorname{cl}(s \cdot$ bin, $\phi)$, then $\psi \in \operatorname{cl}(s, \phi)$;

(IV) if $[\sigma] \psi \in \operatorname{cl}(s, \phi)$, then $\psi \in \operatorname{cl}(s, \phi)$.

\section{Proof:}

(I) By Lemma 3.2(II).

(II) Assume $[\sigma] \psi \in \operatorname{cl}(s, \phi)$. If $\psi \neq[\leq] \varphi$, then $[\sigma][\leq] \psi \in \operatorname{cl}(s, \phi)$ and $[\leq] \psi \in \operatorname{cl}(s, \phi)$. By definition of $\operatorname{cl}(s \cdot f$ in,$\phi)$, we get that both $[\leq] \psi$ and $\psi$ are in $\operatorname{cl}(s \cdot f i n, \phi)$. If $\psi=[\leq] \varphi$, then $[\leq] \varphi \in \operatorname{cl}(s, \phi)$ and by definition of $\operatorname{cl}(s \cdot$ fin,$\phi),[\leq] \varphi \in \operatorname{cl}(s \cdot$ fin,$\phi)$.

(III) Assume $[\sigma] \psi \in \operatorname{cl}(s \cdot \operatorname{bin}, \phi)$. So $[\sigma] \psi \in \operatorname{cl}(s, \phi)$ by Lemma 3.2(I) and therefore $\psi \in \operatorname{cl}(s, \phi)$.

(IV) This is immediate since $\operatorname{cl}(s, \phi)$ is closed.

Lemma 3.8. Let $s \in\{\operatorname{sim}, \text { bin, fin }\}^{*}$ and $\phi$ be a formula.

(I) if $[\sigma] \psi \in \operatorname{cl}(s \cdot \operatorname{sim}, \phi)$, then $\psi \in \operatorname{cl}(s, \phi)$;

(II) if $[\sigma] \psi \in \operatorname{cl}(s \cdot f i n, \phi)$, then $\psi \in \operatorname{cl}(s, \phi)$;

(III) if $[\sigma] \psi \in \operatorname{cl}(s, \phi)$, then $\psi \in \operatorname{cl}(s \cdot b i n, \phi)$;

(IV) if $[\sigma] \psi \in \operatorname{cl}(s, \phi)$, then $\psi \in \operatorname{cl}(s, \phi)$;

\section{Proof:}

(I) This is immediate since $\operatorname{cl}(s \cdot \operatorname{sim}, \phi) \subseteq \operatorname{cl}(s, \phi)$ and $\operatorname{cl}(s, \phi)$ is closed.

(II) This is immediate since $\operatorname{cl}(s \cdot f i n, \phi) \subseteq \operatorname{cl}(s, \phi)$ and $\operatorname{cl}(s, \phi)$ is closed.

(III) Assume $[\sigma] \psi \in \operatorname{cl}(s, \phi)$. If $\psi=[\leq] \psi^{\prime},[\sigma] \psi \in \operatorname{cl}(s \cdot$ bin, $\phi)$ and $\psi \in \operatorname{cl}(s \cdot$ bin, $\phi)$ since $\operatorname{cl}(s \cdot \operatorname{bin}, \phi)$ is closed. Otherwise $\left(\psi \neq[\leq] \psi^{\prime}\right),[\sigma][\leq] \psi \in \operatorname{cl}(s, \phi)$ since $\operatorname{cl}(s, \phi)$ is closed and $[\sigma][\leq] \psi,[\leq] \psi,[\sigma] \psi, \psi \in \operatorname{cl}(s \cdot \operatorname{bin}, \phi)$.

(IV) By Lemma 3.7(IV).

Lemma 3.9. Let $s \in\{\operatorname{sim}, \text { bin, } \text { fin }\}^{*}$ and $\phi$ be a formula.

(I) if $[\leq] \psi \in \operatorname{cl}(s, \phi)$, then $\psi \in \operatorname{cl}(s \cdot$ fin, $\phi)$;

(II) if $[\leq] \psi \in \operatorname{cl}(s \cdot b i n, \phi)$, then $\psi \in \operatorname{cl}(s, \phi)$;

(III) if $[\leq] \psi \in \operatorname{cl}(s, \phi)$, then $\psi \in \operatorname{cl}(s, \phi)$;

\section{Proof:}

(I) By definition since $\operatorname{cl}(s \cdot f i n, \phi)$ is closed.

(II) This is immediate since $\operatorname{cl}(s \cdot \operatorname{bin}, \phi) \subseteq \operatorname{cl}(s, \phi)$ and $\operatorname{cl}(s, \phi)$ is closed.

(III) This is immediate since $\operatorname{cl}(s, \phi)$ is closed.

Lemma 3.10. Let $s \in\{\operatorname{sim}, \text { bin, fin }\}^{*}$ and $\phi$ be a formula.

(I) if $[\geq] \psi \in \operatorname{cl}(s \cdot$ fin, $\phi)$, then $\psi \in \operatorname{cl}(s, \phi)$; 
(II) if $[\geq] \psi \in \operatorname{cl}(s, \phi)$, then $\psi \in \operatorname{cl}(s \cdot b i n, \phi)$;

(III) if $[\geq] \psi \in \operatorname{cl}(s, \phi)$, then $\psi \in \operatorname{cl}(s, \phi)$.

\section{Proof:}

(I) This is immediate since $\operatorname{cl}(s \cdot f i n, \phi) \subseteq \operatorname{cl}(s, \phi)$ and $\operatorname{cl}(s, \phi)$ is closed.

(II) This is immediate since $\operatorname{cl}(s \cdot f i n, \phi)$ is closed.

(III) This is obvious since $\operatorname{cl}(s, \phi)$ is closed.

Lemma 3.11. Let $s \in\{\text { sim, bin, fin }\}^{*}$ and $\phi$ be a formula.

(I) if $[\sigma] \psi \in \operatorname{cl}(s \cdot$ fin, $\phi)$, then $[\sigma] \psi \in \operatorname{cl}(s, \phi)$;

(II) if $[\sigma] \psi \in \operatorname{cl}(s, \phi)$, then $[\sigma] \psi \in \operatorname{cl}(s \cdot b i n, \phi)$;

(III) if $[\sigma] \psi \in \operatorname{cl}(s, \phi)$, then $[\sigma] \psi \in \operatorname{cl}(s, \phi)$.

\section{Proof:}

(I) This is immediate since $\operatorname{cl}(s \cdot$ fin,$\phi) \subseteq \operatorname{cl}(s, \phi)$.

(II) See the proof of Lemma 3.8(III).

(III) Obvious.

Before defining the main algorithm of the paper, let us conclude by presenting some relationships between the relations $\preceq$ and $\approx$ and the relations from the NIL-models.

Lemma 3.12. Let $\mathcal{M}=\left\langle W, R_{\sigma}, R_{\leq}, R_{\geq}, m\right\rangle$ be a NIL-model and $w, w^{\prime} \in W$. Let $s \in\{\text { sim, bin, fin }\}^{*}$, $s^{\prime}, s^{\prime \prime} \in\{\lambda$, sim, fin, bin $\}$ and $\phi$ be a formula. Let

$$
X_{w} \stackrel{\text { def }}{=}\left\{\psi \in \operatorname{cl}\left(s \cdot s^{\prime}, \phi\right): \mathcal{M}, w \models \psi\right\} \quad X_{w^{\prime}} \stackrel{\text { def }}{=}\left\{\psi \in \operatorname{cl}\left(s \cdot s^{\prime \prime}, \phi\right): \mathcal{M}, w^{\prime} \models \psi\right\}
$$

Then,

(I) $X_{w}$ is $s \cdot s^{\prime}$-consistent and $X_{w^{\prime}}$ is $s \cdot s^{\prime \prime}$-consistent;

(II) if $\left\langle s^{\prime}, s^{\prime \prime}\right\rangle \in\{\langle\lambda, \operatorname{sim}\rangle,\langle\operatorname{sim}, \lambda\rangle,\langle\lambda, \lambda\rangle\}$ and $\left\langle w, w^{\prime}\right\rangle \in R_{\sigma}$, then $X_{w} \approx X_{w^{\prime}}$;

(III) if $\left\langle s^{\prime}, s^{\prime \prime}\right\rangle \in\{\langle\lambda$, fin $\rangle,\langle$ bin,$\lambda\rangle,\langle\lambda, \lambda\rangle\}$ and $\left\langle w, w^{\prime}\right\rangle \in R_{\leq}$, then $X_{w} \preceq X_{w^{\prime}}$.

The proof is by an easy verification using the previous lemmas.

\section{NIL is in PSPACE}

\subsection{The algorithm}

In Figure 1, the function $\operatorname{NIL-WORLD}(\Sigma, s, \phi)$ returning a Boolean is defined. $\Sigma$ is a nonempty finite sequence of subsets of $\operatorname{cl}(\{\phi\})$ and $s \in\{\operatorname{sim}, f i n, b i n\}^{*}$. For any $X \subseteq \operatorname{cl}(\{\phi\})$ and for any call $\operatorname{NLL}-\operatorname{WORLD}(\Sigma, s, \phi)$ in $\operatorname{NIL-WORLD}(X, \lambda, \phi)$ (at any recursion depth), we have last $(\Sigma) \subseteq$ $\operatorname{cl}(s, \phi)$. In the next section we shall show that

- $|\Sigma| \leq 25 \times|\phi|^{2}$; 
function NIL-WORLD $(\Sigma, s, \phi)$

if $\operatorname{last}(\Sigma)$ is not $s$-consistent, then return false;

for $[\sigma] \psi \in \operatorname{cl}(s, \phi) \backslash \operatorname{last}(\Sigma)$ do

for each $X_{\psi} \subseteq \operatorname{cl}(s \cdot \operatorname{sim}, \phi) \backslash\{\psi\}$ such that $\operatorname{last}(\Sigma) \approx X_{\psi}$, call $\operatorname{NIL-WORLD}\left(X_{\psi}, s\right.$. sim, $\phi)$. If all these calls return false, then return false;

for $[\leq] \psi \in \operatorname{cl}(s, \phi) \backslash \operatorname{last}(\Sigma)$ do

if there is no $X \in \Sigma$ such that $\psi \notin X$, last $(\Sigma) \preceq X$, and last $(s)=$ fin, then for each $X_{\psi} \subseteq \operatorname{cl}(s \cdot$ fin,$\phi) \backslash\{\psi\}$ such that last $(\Sigma) \preceq X_{\psi}$, if last $(s)=$ fin, then call $\operatorname{NIL-WORLD}\left(\Sigma \cdot X_{\psi}, s, \phi\right)$, otherwise call NIL-WORLD $\left(X_{\psi}, s \cdot f i n, \phi\right)$. If all these calls return false, then return false;

for $[\geq] \psi \in \operatorname{cl}(s, \phi) \backslash \operatorname{last}(\Sigma)$ do

if there is no $X \in \Sigma$ such that $\psi \notin X$, last $(\Sigma) \succeq X$, and last $(s)=\operatorname{bin}$, then for each $X_{\psi} \subseteq \operatorname{cl}(s \cdot \operatorname{bin}, \phi) \backslash\{\psi\}$ such that $X_{\psi} \preceq \operatorname{last}(\Sigma)$, if last $(s)=$ bin, then call $\operatorname{NIL-WORLD}\left(\Sigma \cdot X_{\psi}, s, \phi\right)$, otherwise call NIL-WORLD $\left(X_{\psi}, s \cdot b i n, \phi\right)$. If all these calls return false, then return false;

Return true.

Figure 1. Algorithm NIL-WORLD

- $|s| \leq 3 \times|\phi|$.

The function NIL-WORLD is actually defined on the model of the function K-WORLD in [20] and it is an extension of the function $S 4_{t}$-WORLD defined in [31] (see also [10, 22, 35]). The results given in Section 3 are crucial to guarantee that NIL-WORLD is correct and terminates. By a successful call of NIL-WORLD $(\Sigma, s, \phi)$ we mean that it returns true.

\subsection{Termination and complexity upper bounds}

If NIL-WORLD is correct, then for any formula $\phi, \phi$ is NIL satisfiable iff there is $X \subseteq \operatorname{cl}(\{\phi\})$ such that $\phi \in X$ and NIL-WORLD $(X, \lambda, \phi)$ returns true. Each subset $X \subseteq \operatorname{cl}(\{\phi\})$ can be represented as a bitstring of length $5 \times|\phi|$ since $\operatorname{card}(\operatorname{cl}(\phi))<5 \times|\phi|$. At each level of the recursion, we use space in $\mathcal{O}(|\phi|)$ by implementing $\Sigma$ as a global stack. For instance, in the parts of NIL-WORLD of the form "for each $X_{\psi}$...then return false", the implementation uses a bitstring of length $5 \times|\phi|$ to encode $X_{\psi}$ and a Boolean indicating whether a call has returned true.

Suppose that in the call of $\operatorname{NIL-WORLD}(X, \lambda, \phi)$ at some recursion depth, NIL-WORLD $(\Sigma, s, \phi)$ is called with $\Sigma=X_{1} \cdot \ldots \cdot X_{n}, n \geq 2$. We treat the case $s=s^{\prime} \cdot$ fin and the case $s=s^{\prime} \cdot$ bin 
is omitted because it is very similar. Moreover, if $s=s^{\prime} \cdot \operatorname{sim}$, then $|\Sigma|=1$ and hence this case is not relevant, since we assume $n \geq 2$. For $i \in\{1, \ldots, n-1\},[\leq] \psi \in X_{i}$ implies $[\leq] \psi \in X_{i+1}$. Thus, $\Sigma$ can be written as $\Sigma=\Sigma_{1} \cdot \ldots \cdot \Sigma_{n^{\prime}}$ where for $i, i^{\prime} \in\left\{1, \ldots, n^{\prime}\right\}$

- the elements of $\Sigma_{i}$ contain the same $[\leq]$-formulae;

- $i<i^{\prime}$ implies the set of $[\leq]$-formulae of $\Sigma_{i}$ is a proper subset of the set of $[\leq]$-formulae of $\Sigma_{i^{\prime}}$

Since there are less than $5 \times|\phi|[\leq]$-formulae in $\operatorname{cl}(\{\phi\}), n^{\prime} \leq 5 \times|\phi|$.

Let $i \in\left\{1, \ldots, n^{\prime}\right\} . \Sigma_{i}$ can be written as $\Sigma_{i}=\Sigma_{i}^{1} \cdot \ldots \cdot \Sigma_{i}^{l(i)}$ where for $j, j^{\prime} \in\{1, \ldots, l(i)\}$, the elements of $\Sigma_{i}^{j}$ contains the same $[\geq]$-formulae and $[\sigma]$-formulae and $j<j^{\prime}$ implies the set of $[\geq]$-formulae and $[\sigma]$-formulae of $\Sigma_{i}^{j^{\prime}}$ is a proper subset of the set of $[\geq]$-formulae and $[\sigma]$-formulae of $\Sigma_{i}^{j}$.

For $i \in\{1, \ldots, n\}$, let $Z_{i}^{1} \stackrel{\text { def }}{=}\left\{[\geq] \psi:[\geq] \psi \in X_{i}\right\}$ and $Z_{i}^{2} \stackrel{\text { def }}{=}\left\{[\sigma] \psi:[\sigma] \psi \in X_{i}\right\}$. We have

$$
\left(Z_{n}^{1} \cup Z_{n}^{2}\right) \subseteq \ldots \subseteq\left(Z_{1}^{1} \cup Z_{1}^{2}\right)
$$

One can see that $l(1)+\ldots+l\left(n^{\prime}\right)$ is in $\mathcal{O}(|\phi|)$, since the above sequence contains at most $\mathcal{O}(|\phi|)$ proper inclusions. Actually, $l(1)+\ldots+l\left(n^{\prime}\right)<5 \times|\phi|$.

Now let us estimate the maximal length $\left|\Sigma_{i}^{j}\right|$ for $i \in\left\{1, \ldots, n^{\prime}\right\}$ and $j \in\{1, \ldots, l(i)\}$. Suppose $\Sigma_{i}^{j}=Y_{1} \cdot \ldots \cdot Y_{k}$. If $1 \leq u<u^{\prime} \leq k$, then for $[\leq] \psi \in Y_{u}$, we have $[\leq] \psi \in Y_{u^{\prime}}$. Since for $2 \leq \alpha<k$, $\operatorname{NIL-WORLD}\left(\Sigma_{1} \ldots \Sigma_{i-1} Y_{1} \cdot \ldots \cdot Y_{\alpha}, s, \phi\right)$ calls NIL-WORLD $\left(\Sigma_{1} \ldots \Sigma_{i-1} Y_{1} \cdot \ldots \cdot Y_{\alpha+1}, s, \phi\right)$, there are formulae $\psi_{2}, \ldots, \psi_{k}$ in $\operatorname{cl}(\{\phi\})$ such that $\psi_{\alpha} \notin Y_{\alpha}$ and for $1 \leq \alpha^{\prime}<\alpha, \psi_{\alpha} \in Y_{\alpha^{\prime}}$. Hence $\psi_{2}, \ldots, \psi_{k}$ are $(k-1)$ different formulae in $\operatorname{cl}(\{\phi\})$. Hence $k$ is in $\mathcal{O}(|\phi|)$. More precisely, $k<5 \times|\phi|$. So the maximal length of $\Sigma$ is in $\mathcal{O}\left(|\phi|^{2}\right)$. More precisely, $|\Sigma| \leq 25 \times|\phi|^{2}$. Termination is not yet proved since there are moments in the computation where the length of $\Sigma$ strictly decreases. However, the following observations will help finishing the proof of termination of NIL-WORLD $(X, \lambda, \phi)$ :

- If NIL-WORLD $(\Sigma, s, \phi)$ calls NIL-WORLD $\left(\Sigma^{\prime}, s^{\prime}, \phi\right)$ (not at any recursion depth), then either $|\Sigma|<\left|\Sigma^{\prime}\right|$ or $|s|<\left|s^{\prime}\right|$

- Any call $\operatorname{NLL}-\operatorname{WORLD}(\Sigma, s, \phi)$ from $\operatorname{NIL-WORLD}(X, \lambda, \phi)$ satisfies that neither bin bin nor fin fin is a substring of $s$;

- Any call NIL-WORLD $(\Sigma, s, \phi)$ with $|s| \geq 3 \times|\phi|$ does not call recursively NIL-WORLD. This means that no more recursive calls to NIL-WORLD is executed (see Theorem 3.1).

Consequently, the depth of the recursion is in $\mathcal{O}\left(|\phi|^{3}\right)$. More precisely, the depth is bounded by $75 \times|\phi|^{3}$. Since we need space in $\mathcal{O}(|\phi|)$ at each level of the recursion, the total space to compute $\operatorname{NIL}-\operatorname{WORLD}(X, \lambda, \phi)$ for $X \subseteq \operatorname{cl}(\{\phi\})$ is in $\mathcal{O}\left(|\phi|^{4}\right)$. As a consequence we have,

Theorem 4.1. Let $X \subseteq \operatorname{cl}(\{\phi\})$.

(I) $\operatorname{NIL-WORLD}(X, \lambda, \phi)$ terminates and requires space in $\mathcal{O}\left(|\phi|^{4}\right)$;

(II) Let $\operatorname{NIL-WORLD}(\Sigma, s, \phi)$ be a call in the computation of $\operatorname{NIL-WORLD}(X, \lambda, \phi)$. Then, $|\Sigma| \leq$ $25 \times|\phi|^{2}$ and $|s| \leq 3 \times|\phi|$; 
(III) Let a call NIL-WORLD $\left(\Sigma^{\prime}, s^{\prime}, \phi\right)$ be made in $\operatorname{NIL-WORLD}(\Sigma, s, \phi)$ in the computation of $\operatorname{NIL}-\operatorname{WORLD}(X, \lambda, \phi)$. Then,

$$
\left\langle 3 \times|\phi|-\left|s^{\prime}\right|, 25 \times|\phi|^{2}-\left|\Sigma^{\prime}\right|\right\rangle\left\langle\left\langle 3 \times|\phi|-|s|, 25 \times|\phi|^{2}-|\Sigma|\right\rangle\right.
$$

where $<$ is the standard (well-founded) lexicographical ordering on $\omega^{2}$.

\subsection{Correction}

Theorem 4.1 is an important step to prove that NIL satisfiability is in PSPACE but it is not sufficient. Indeed, up to now we have no guarantee that the function NIL-WORLD is correct. This is shown in the next two lemmas.

Lemma 4.1. Let $\phi$ be a NIL-formula and let $Y \subseteq \operatorname{cl}(\{\phi\})$ satisfy $\phi \in Y$. If $\operatorname{NIL-WORLD}(Y, \lambda, \phi)$ returns true, then $\phi$ is NIL-satisfiable.

\section{Proof:}

Assume that NIL-WORLD $(Y, \lambda, \phi)$ returns true. Let us build a NIL-model $\mathcal{M}=\left\langle W, R_{\leq}, R_{\geq}, R_{\sigma}, m\right\rangle$ for which there is $w \in W$ such that for all $\psi \in \operatorname{cl}(\{\phi\}), \mathcal{M}, w \models \psi$ iff $\psi \in Y$.

Let $S$ be the set of strings $s$ over $\{$ sim, bin, fin $\}$ such that $|s| \leq 3 \times|\phi|$. We define $W$ as the set of pairs $\langle X, s\rangle$ for which there is a finite sequence $\left\langle\Sigma_{1}, s_{1}\right\rangle, \ldots,\left\langle\Sigma_{k}, s_{k}\right\rangle(k \geq 1)$ such that

1. for $i \in\{1, \ldots, k\}, \operatorname{NIL-WORLD}\left(\Sigma_{i}, s_{i}, \phi\right)$ is called in NIL-WORLD $(Y, \lambda, \phi)$ (at any depth of the recursion);

2. $\Sigma_{1}=Y ; s_{1}=\lambda ; \operatorname{last}\left(\Sigma_{k}\right)=X ; s_{k}=s ;$

3. for $i \in\{1, \ldots, k\}, \operatorname{NIL-WORLD}\left(\Sigma_{i}, s_{i}, \phi\right)$ returns true;

4. for $i \in\{1, \ldots, k-1\}, \operatorname{NIL}-\operatorname{WORLD}\left(\Sigma_{i}, s_{i}, \phi\right)$ calls directly $\operatorname{NIL-WORLD}\left(\Sigma_{i+1}, s_{i+1}, \phi\right)$.

The conditions 3. and 4. state that we only want to record those pairs $\langle X, s\rangle \in \operatorname{clos} \times S$ that contribute to making NIL-WORLD $(Y, \lambda, \phi)$ true. $\langle Y, \lambda\rangle \in W$ by assumption. Furthermore, for all $\langle X, s\rangle \in W, X$ is $s$-consistent.

Let us define the binary relation $R_{\text {sim }}$ on $W$ as follows: $\langle X, s\rangle R_{s i m}\left\langle X^{\prime}, s^{\prime}\right\rangle \stackrel{\text { def }}{\Leftrightarrow}$ there is a successful call NIL-WORLD $(\Sigma, s, \phi)$ in $\operatorname{NIL-WORLD}(Y, \lambda, \phi)$ (at any depth of the recursion) such that

1. $\operatorname{last}(\Sigma)=X$;

2. NIL-WORLD $(\Sigma, s, \phi)$ calls NIL-WORLD $\left(\Sigma^{\prime}, s^{\prime}, \phi\right)$ successfully in the "sim" segment of $\operatorname{NIL-WORLD}(\Sigma, s, \phi)$;

3. $\operatorname{last}\left(\Sigma^{\prime}\right)=X^{\prime}$.

If $\langle X, s\rangle R_{\text {sim }}\left\langle X^{\prime}, s^{\prime}\right\rangle$, then $s^{\prime}=s \cdot \operatorname{sim}, \Sigma^{\prime}=X^{\prime}$ and $X \approx X^{\prime}$.

In a similar way, let us define the binary relation $R_{f i n}$ [resp. $\left.R_{b i n}\right]$ on $W$ as follows: $\langle X, s\rangle R_{\text {fin }}\left\langle X^{\prime}, s^{\prime}\right\rangle$ [resp. $\left.\langle X, s\rangle R_{b i n}\left\langle X^{\prime}, s^{\prime}\right\rangle\right] \stackrel{\text { def }}{\Leftrightarrow}$ there is a successful call of NIL-WORLD $(\Sigma, s, \phi)$ in $\operatorname{NIL-WORLD}(Y, \lambda, \phi)$ (at any depth of the recursion) such that 
1. either

(a) $\operatorname{last}(\Sigma)=X$;

(b) $\operatorname{NIL-WORLD}(\Sigma, s, \phi)$ calls NIL-WORLD $\left(\Sigma^{\prime}, s^{\prime}, \phi\right)$ successfully in the "fin" [resp. "bin"] segment of NIL-WORLD $(\Sigma, s, \phi)$;

(c) $\operatorname{last}\left(\Sigma^{\prime}\right)=X^{\prime}$.

2. or there is a finite sequence $\left\langle\Sigma_{1}, s_{1}\right\rangle, \ldots,\left\langle\Sigma_{k}, s_{k}\right\rangle$ such that:

(a) $\operatorname{last}\left(\Sigma_{k}\right)=X ; \operatorname{last}\left(\Sigma_{1}\right)=X^{\prime}$;

(b) $\Sigma_{k}=\Sigma ; s_{k}=s ; s_{1}=s^{\prime}$;

(c) for $i \in\{1, \ldots, k\}$, $\left\langle\operatorname{last}\left(\Sigma_{i}\right), s_{i}\right\rangle \in W$;

(d) for $i \in\{1, \ldots, k-1\}, \operatorname{NIL-WORLD}\left(\Sigma_{i}, s_{i}, \phi\right)$ calls NIL-WORLD $\left(\Sigma_{i+1}, s_{i+1}, \phi\right)$ in the "fin" [resp. "bin"] segment of NIL-WORLD and both NIL-WORLD $\left(\Sigma_{i}, s_{i}, \phi\right)$ and $\operatorname{NIL}-\operatorname{WORLD}\left(\Sigma_{i+1}, s_{i+1}, \phi\right)$ return true;

(e) the call NIL-WORLD $\left(\Sigma_{k}, s_{k}, \phi\right)$ enters in the "fin" [resp. "bin"] segment of NIL-WORLD, $\operatorname{last}\left(s_{k}\right)=\operatorname{fin}\left[\right.$ resp. $\left.\operatorname{last}\left(s_{k}\right)=\operatorname{bin}\right]$, and for some formula $[\leq] \psi \in \operatorname{cl}(s, \phi) \backslash X$ [resp. $[\geq] \psi \in \operatorname{cl}(s, \phi) \backslash X], \psi \notin X^{\prime}$ and $X \preceq X^{\prime}\left[\right.$ resp. $X \succeq X^{\prime}$.

The definition of $\mathcal{M}$ can be now completed:

- $R_{\leq} \stackrel{\text { def }}{=}\left(R_{f i n} \cup R_{b i n}^{-1}\right)^{*}$;

- $R_{\geq} \stackrel{\text { def }}{=}\left(R_{b i n} \cup R_{f i n}^{-1}\right)^{*}$;

- $R_{\sigma} \stackrel{\text { def }}{=} R_{\geq} \circ\left(R_{\text {sim }} \cup R_{\text {sim }}^{-1} \cup\{\langle\langle X, s\rangle,\langle X, s\rangle\rangle:\langle X, s\rangle \in W\}\right) \circ R_{\leq}$;

- for $\mathrm{p} \in \mathrm{For}_{0}, m(\mathrm{p}) \stackrel{\text { def }}{=}\{\langle X, s\rangle \in W: \mathrm{p} \in X\}$.

It is easy to see that $R_{\leq}$and $R_{\geq}$are reflexive and transitive relations and $R_{\geq}$is the converse of $R_{\leq}$. Moreover, it is easy to show that $R_{\sigma}$ is reflexive and symmetric and $R_{\geq} \circ R_{\sigma} \circ R_{\leq} \subseteq R_{\sigma}$. So, $\mathcal{M}$ is NIL-model and $W$ is of cardinality $2^{\mathcal{O}(|\phi|)}$. One can show:

(i) $\langle X, s\rangle\left(R_{f i n} \cup R_{b i n}^{-1}\right)\left\langle X^{\prime}, s^{\prime}\right\rangle$ implies $X \preceq X^{\prime}$;

(ii) $\langle X, s\rangle\left(R_{b i n} \cup R_{f i n}^{-1}\right)\left\langle X^{\prime}, s^{\prime}\right\rangle$ implies $X^{\prime} \preceq X$;

(iii) $\langle X, s\rangle\left(R_{\text {sim }} \cup R_{\text {sim }}^{-1} \cup\{\langle\langle X, s\rangle,\langle X, s\rangle\rangle:\langle X, s\rangle \in W\}\right)\left\langle X^{\prime}, s^{\prime}\right\rangle$ implies $X \approx X^{\prime}$.

So,

(iv) $\langle X, s\rangle R_{\leq}\left\langle X^{\prime}, s^{\prime}\right\rangle$ implies for all $[\leq] \psi \in X, \psi \in X^{\prime}$ (by definition of $\preceq$ );

(v) $\langle X, s\rangle R_{\geq}\left\langle X^{\prime}, s^{\prime}\right\rangle$ implies for all $[\geq] \psi \in X, \psi \in X^{\prime}$ (by definition of $\preceq$ );

(vi) $\langle X, s\rangle R_{\sigma}\left\langle X^{\prime}, s^{\prime}\right\rangle$ implies for all $[\sigma] \psi \in X, \psi \in X^{\prime}$ (by definition of $\approx$ and by Lemma 3.6(I)). 
By induction on the structure of $\psi$ we shall show that for all $\langle X, s\rangle \in W$, for all $\psi \in \operatorname{cl}(s, \phi)$, $\psi \in X$ iff $\mathcal{M},\langle X, s\rangle \models \psi$. The case when $\psi$ is a propositional variable is by definition of $m$.

Induction hypothesis: for all $\psi \in \operatorname{cl}(\{\phi\})$ such that $|\psi| \leq n$, for all $\langle X, s\rangle \in W$, if $\psi \in \operatorname{cl}(s, \phi)$, then $\psi \in X$ iff $\mathcal{M},\langle X, s\rangle \models \psi$.

Let $\psi$ be a formula in $\operatorname{cl}(\{\phi\})$ such that $|\psi| \leq n+1$. The cases when the outermost connective of $\psi$ is Boolean is a consequence of the $s$-consistency of $X$ and the induction hypothesis. Let us treat the other cases.

Case 1: $\psi=[\leq] \psi^{\prime}$.

Let $\langle X, s\rangle \in W$ such that $\psi \in \operatorname{cl}(s, \phi)$. By definition of $W$, there is a sequence $\Sigma$ such that last $(\Sigma)=X$ and $\operatorname{NIL-WORLD}(\Sigma, s, \phi)$ returns true. If $\psi \notin X$ one of the following two cases occurs.

Case 1.1: there is $X^{\prime}$ in $\Sigma$ such that $X \preceq X^{\prime}, \psi^{\prime} \notin X^{\prime}$ and last $(s)=$ fin. By definition of $W$, there is a subsequence $\Sigma^{\prime}$ of $\Sigma$ and $s^{\prime}$ such that last $\left(\Sigma^{\prime}\right)=X^{\prime}$ and $\operatorname{NIL-WORLD}\left(\Sigma^{\prime}, s^{\prime}, \phi\right)$ returns true (see the conditions 3. and 4. defining $W$ ). Hence, we have $\langle X, s\rangle R_{f i n}\left\langle X^{\prime}, s^{\prime}\right\rangle$ and therefore $\langle X, s\rangle R_{\leq}\left\langle X^{\prime}, s^{\prime}\right\rangle$. Observe that either $s^{\prime}=s$ or $s=s^{\prime} \cdot$ fin. By Lemma 3.9, $\psi^{\prime} \in \operatorname{cl}\left(s^{\prime}, \phi\right)$. By induction hypothesis, $\mathcal{M},\left\langle X^{\prime}, s^{\prime}\right\rangle \not \psi^{\prime}$ and therefore $\mathcal{M},\langle X, s\rangle \not \models \psi$.

Case 1.2: $\operatorname{NLL-WORLD}(\Sigma, s, \phi)$ calls successfully $\operatorname{NIL-WORLD}\left(\Sigma^{\prime}, s^{\prime}, \phi\right)$ in the "fin" segment of NIL-WORLD, last $\left(\Sigma^{\prime}\right)=X^{\prime}, \psi^{\prime} \notin \operatorname{last}\left(\Sigma^{\prime}\right), X \preceq X^{\prime}$ and $X^{\prime} \subseteq \operatorname{cl}\left(s^{\prime}, \phi\right)$. Moreover, we have either $s^{\prime}=s$ or $s^{\prime}=s \cdot$ fin. This is so since $\operatorname{NIL-WORLD}(\Sigma, s, \phi)$ returns true. By definition of $R_{f i n},\langle X, s\rangle R_{f i n}\left\langle X^{\prime}, s^{\prime}\right\rangle$. Furthermore, $\psi^{\prime} \in \mathrm{cl}\left(s^{\prime}, \phi\right)$ by Lemma 3.9. By the induction hypothesis, $\mathcal{M},\left\langle X^{\prime}, s^{\prime}\right\rangle \not \psi^{\prime}$ and therefore $\mathcal{M},\langle X, s\rangle \not \models \psi$.

If $\psi \in X$, then by (iv), for all $\left\langle X^{\prime}, s^{\prime}\right\rangle \in R_{\leq}(\langle X, s\rangle), \psi^{\prime} \in X^{\prime}$ (and $\psi^{\prime} \in \operatorname{cl}\left(s^{\prime}, \phi\right)$ by Lemma 3.9). By the induction hypothesis, $\mathcal{M},\left\langle X^{\prime}, s^{\prime}\right\rangle \models \psi^{\prime}$ and therefore $\mathcal{M},\langle X, s\rangle \models \psi$.

Case 2: $\psi=[\geq] \psi^{\prime}$.

This is analogous to the Case 1 and can be proved by using (v) above and Lemma 3.10.

Case 3: $\psi=[\sigma] \psi^{\prime}$.

By definition of $W$, there is a sequence $\Sigma$ such that last $(\Sigma)=X$ and $\operatorname{NIL-WORLD}(\Sigma, s, \phi)$ returns true. If $\psi \notin X$, then $\operatorname{NIL-WORLD}(\Sigma, s, \phi)$ calls $\operatorname{NIL-WORLD}\left(X^{\prime}, s \cdot \operatorname{sim}, \phi\right)$ in its "sim" segment, $\psi^{\prime} \notin \operatorname{last}\left(X^{\prime}\right), X \approx X^{\prime}$ and $X^{\prime} \subseteq \operatorname{cl}\left(s^{\prime}, \phi\right)$. This is so since $\operatorname{NIL-WORLD}(\Sigma, s, \phi)$ returns true. By definition of $R_{\text {sim }},\langle X, s\rangle R_{\text {sim }}\left\langle X^{\prime}, s \cdot \operatorname{sim}\right\rangle$. By Lemma 3.7, $\psi^{\prime} \in \operatorname{cl}(s \cdot \operatorname{sim}, \phi)$. By the induction hypothesis, $\mathcal{M},\left\langle X^{\prime}, s \cdot \operatorname{sim}\right\rangle \not \psi^{\prime}$ and therefore $\mathcal{M},\langle X, s\rangle \not \psi \psi$.

If $\psi \in X$, by (vi), for all $\left\langle X^{\prime}, s^{\prime}\right\rangle \in R_{\sigma}(\langle X, s\rangle), \psi^{\prime} \in X^{\prime}$ (and $\psi^{\prime} \in \operatorname{cl}\left(s^{\prime}, \phi\right)$ by Lemma 3.7 and Lemma 3.8). By the induction hypothesis, $\mathcal{M},\left\langle X^{\prime}, s^{\prime}\right\rangle \models \psi^{\prime}$ and therefore $\mathcal{M},\langle X, s\rangle \models \psi$.

As a conclusion, since $\phi \in Y$ and $\operatorname{NIL}-\operatorname{WORLD}(Y, \lambda, \phi)$ returns true, $\mathcal{M},\langle Y, \lambda\rangle \models \phi$ and therefore $\phi$ is NIL-satisfiable.

The proof of Lemma 4.1 can be viewed as a way to transform a successful call of NIL-WORLD $(Y, \lambda, \phi)$ into a quasi NIL-model by analyzing the computation tree of $\operatorname{NIL-WORLD}(Y, \lambda, \phi)$. Then, this quasi NIL-model is appropriately completed in order to get a NIL-model. The idea to construct a (standard) model from different coherent pieces is very common to establish decidability and complexity results for modal logics (see e.g. [20, 29, 21, 4]). 
Lemma 4.2. Let $\phi$ be a NIL-formula. If $\phi$ is NIL-satisfiable, then there is $Y \subseteq \operatorname{cl}(\{\phi\})$ such that $\phi \in Y$ and $\operatorname{NLL}-W O R L D(Y, \lambda, \phi)$ returns true.

\section{Proof:}

Assume $\phi$ is NIL-satisfiable. So, there is a NIL-model $\mathcal{M}^{0}=\left\langle W^{0}, R_{\leq}^{0}, R_{\geq}^{0}, R_{\sigma}^{0}, m^{0}\right\rangle$ and $w^{0} \in W^{0}$ such that $\mathcal{M}^{0}, w^{0} \models \phi$. We shall show that

(i) for any $s \in\{\operatorname{sim}, \text { fin, bin }\}^{*}$ such that neither bin $\cdot$ bin nor fin fin occurs in $s$, if $\Sigma$ is a finite nonempty sequence of subsets of $\operatorname{cl}(s, \phi)$ that contains no duplicates, last $(\Sigma)=X$ and there is a NIL-model $\mathcal{M}=\left\langle W, R_{\leq}, R_{\geq}, R_{\sigma}, m\right\rangle$ and $w \in W$ satisfying for all $\psi \in \operatorname{cl}(s, \phi)$, $\mathcal{M}, w \models \psi$ iff $\psi \in X$, then $\operatorname{NIL-WORLD}(\Sigma, s, \phi)$ returns true.

Consequently, by taking $s=\lambda, X=\left\{\psi \in \operatorname{cl}(\{\phi\}): \mathcal{M}^{0}, w^{0} \models \psi\right\}$ and $\Sigma=X$ we get that $\operatorname{NIL}-\operatorname{WORLD}(X, \lambda, \phi)$ returns true.

The proof of (i) is by double induction on the length of $s$ and on the length of $\Sigma$.

Base case 1: $|s|>3 \times|\phi|$.

Then $\operatorname{cl}(s, \phi)=\emptyset$ and therefore (i) holds.

Induction hypothesis 1: for any $s^{\prime} \in\{\operatorname{sim} \text {, fin, bin }\}^{*}$ such that neither bin bin nor fin $\cdot$ fin occurs in $s^{\prime},\left|s^{\prime}\right| \geq n$ for some $n \geq 1$, if $\Sigma$ is a finite nonempty sequence of subsets of $\operatorname{cl}(s, \phi)$ that contains no duplicates, last $(\Sigma)=X$ and there is a NIL-model $\mathcal{M}=\left\langle W, R_{\leq}, R_{\geq}, R_{\sigma}, m\right\rangle$ and $w \in W$ satisfying for all $\psi \in \operatorname{cl}\left(s^{\prime}, \phi\right), \mathcal{M}, w \models \psi$ iff $\psi \in X$, then $\operatorname{NIL-WORLD}\left(\Sigma, s^{\prime}, \phi\right)$ returns true.

Let $s \in\{\operatorname{sim}, \text { fin, bin }\}^{*}$ such that neither bin.bin nor fin f fin occurs in $s,|s|=n-1$. Let $\Sigma$ be a finite nonempty sequence of subsets of $\operatorname{cl}(s, \phi)$ that contains no duplicates with $\operatorname{last}(\Sigma)=X$. and $\mathcal{M}$ be a NIL-model $\mathcal{M}=\left\langle W, R_{\leq}, R_{\geq}, R_{\sigma}, m\right\rangle$ with $w \in W$ satisfying for all $\psi \in \operatorname{cl}(s, \phi)$, $\mathcal{M}, w \models \psi$ iff $\psi \in X$. Now we use a second induction on the length of $\Sigma$.

Base case 2: $|\Sigma|>2^{\operatorname{card}(\mathrm{cl}(s, \phi))}$.

$\Sigma$ contains duplicates and (i) holds.

Induction hypothesis 2: for all finite nonempty sequence $\Sigma$ of subsets of $\operatorname{cl}(s, \phi)$ that contains no duplicates with $\operatorname{last}(\Sigma)=X,|\Sigma| \geq n^{\prime}$ for some $n^{\prime} \geq 1$ and there is a NIL-model $\mathcal{M}=$ $\left\langle W, R_{\leq}, R_{\geq}, R_{\sigma}, m\right\rangle$ and $w \in W$ satisfying for all $\psi \in \operatorname{cl}(s, \phi), \mathcal{M}, w \models \psi$ iff $\psi \in X$, then $\operatorname{NIL-WORLD}(\Sigma, s, \phi)$ returns true.

Assume $\Sigma$ is a sequence of subsets of $\operatorname{cl}(s, \phi)$ that contains no duplicates, last $(\Sigma)=X,|\Sigma|=n^{\prime}-1$ and there is a NIL-model $\mathcal{M}=\left\langle W, R_{\leq}, R_{\geq}, R_{\sigma}, m\right\rangle$ and $w \in W$ satisfying for all $\psi \in \operatorname{cl}(s, \phi)$, $\mathcal{M}, w \models \psi$ iff $\psi \in X$. Consequently, $X$ is $s$-consistent by Lemma 3.5. So NIL-WORLD $(\Sigma, s, \phi)$ returns false only because either the segment "sim" or the segment "bin" or the segment "fin" returns false.

Case 1: Consider $[\leq] \psi \in \operatorname{cl}(s, \phi) \backslash X$ such that $\mathcal{M}, w \not \models[\leq] \psi$.

There is $w^{\prime} \in W$ such that $\left\langle w, w^{\prime}\right\rangle \in R_{\leq}$and $\mathcal{M}, w^{\prime} \not \models \psi$. Let $Y$ be the subset of $\operatorname{cl}(s \cdot$ fin, $\phi)$ such that for $\varphi \in \operatorname{cl}(s \cdot$ fin,$\phi), \varphi \in Y \stackrel{\text { def }}{\Leftrightarrow} \mathcal{M}, w^{\prime} \models \varphi$. So, $\psi \notin Y$ and $X \preceq Y$ by Lemma 3.12 (III).

If $Y \in \Sigma$ and last $(s)=$ fin, then no recursive call to NIL-WORLD is needed by definition of NIL-WORLD. In the case when either $Y \notin \Sigma$ or last $(s) \neq f i n$, by induction hypothesis, either 
NIL-WORLD $(\Sigma \cdot Y, s, \phi)$ returns true (by the induction hypothesis 2 ) or NIL-WORLD $(Y, s \cdot f i n, \phi)$ returns true (by the induction hypothesis 1 ). Therefore, $\operatorname{NIL-WORLD}(\Sigma, s, \phi)$ does not return false in the "fin" segment of NIL-WORLD. Similarly, we can show that NIL-WORLD $(\Sigma, s, \phi)$ does not return false in the "bin" segment of NIL-WORLD.

Case 2: consider $[\sigma] \psi \in \operatorname{cl}(s, \phi) \backslash X$ such that $\mathcal{M}, w \not \models[\sigma] \psi$.

There is $w^{\prime} \in W$ such that $\left\langle w, w^{\prime}\right\rangle \in R_{\sigma}$ and $\mathcal{M}, w^{\prime} \not \models \psi$. Let $Y$ be the subset of $\operatorname{cl}(s \cdot \operatorname{sim}, \phi)$ such that for $\varphi \in \operatorname{cl}(s \cdot \operatorname{sim}, \phi), \varphi \in Y \stackrel{\text { def }}{\Leftrightarrow} \mathcal{M}, w^{\prime} \models \varphi$. So, $\psi \notin Y$ and $X \approx Y$ by Lemma 3.12(II). By the induction hypothesis 1 (remember $\psi \in \operatorname{cl}(s \cdot \operatorname{sim}, \phi)$ ), NIL-WORLD $(Y, s \cdot \operatorname{sim}, \phi)$ returns true. Therefore, NIL-WORLD $(\Sigma, s, \phi)$ does not return false in the "sim" segment of NIL-WORLD. The fact that the induction hypothesis 2 is not used in this case should not come as a surprise. Indeed, NIL-WORLD $\left(\Sigma^{\prime \prime}, s^{\prime \prime} \cdot \operatorname{sim}, \phi\right)=\operatorname{NIL-WORLD}\left(\operatorname{last}\left(\Sigma^{\prime \prime}\right), s^{\prime \prime} \cdot \operatorname{sim}, \phi\right)$ for any nonempty finite sequence $\Sigma^{\prime \prime}$ of elements of $\mathcal{P}\left(\operatorname{cl}\left(s^{\prime \prime} \cdot \operatorname{sim}, \phi\right)\right)$ and $s^{\prime \prime} \in\{\operatorname{sim}, \text { fin, bin }\}^{*}$. In other words, for a 'sim' transition, we do not need to keep track of the history of the path. Actually, we only need to check the predecessor which is done with the syntactic relation $\approx$.

Consequently, since neither the segment "sim" nor the segment "bin" nor the segment "fin" returns false, NIL-WORLD $(\Sigma, s, \phi)$ returns true and this completes the proof.

Since NIL-WORLD is correct, the proof of Lemma 4.1 provides the finite model property for NIL and an exponential bound for the size of the models exists. These results could be also obtained via a filtration construction but here we get them as a by-product of the complexity result.

Finally,

Theorem 4.2. NIL satisfiability is in PSPACE.

\section{Proof:}

By Lemma 4.1 and Lemma 4.2, for any formula $\phi, \phi$ is NIL-satisfiable iff there is $X \subseteq \operatorname{cl}(\{\phi\})$ such that $\operatorname{NIL-WORLD}(X, \lambda, \phi)$ returns true. By Theorem $4.1, \operatorname{NIL}-\operatorname{WORLD}(X, \lambda, \phi)$ requires space in $\mathcal{O}\left(|\phi|^{4}\right)$ and the index necessary to remember which $X \subseteq \operatorname{cl}(\{\phi\})$ have been already treated, is in space $\mathcal{O}(\log \operatorname{card}(\mathcal{P}(\operatorname{cl}(\{\phi\}))))$, that is in space $\mathcal{O}(|\phi|)$.

\section{Concluding remarks}

We have shown that the information logic NIL introduced in [25] and further studied in [32] has a PSPACE-complete satisfiability problem. This should not come as a real surprise since PSPACE is known to be the complexity class for modal logics. However, it is also known that the satisfiability of polymodal logics can be EXPTIME-hard as soon as the universal modality or the reflexive transitive modality is added to appropriate modal logics [17]. For instance, the bimodal logic (say, with modal operators [1] and [2]) characterized by the class of frames $\left\langle W, R_{1}, R_{2}\right\rangle$ such that $R_{1} \subseteq R_{2} \subseteq W \times W$ and $R_{2}$ is an equivalence relation is EXPTIMEhard [17]. Remember that in a NIL model we also have $R_{\leq} \subseteq R_{\sigma}$ and $R_{\geq} \subseteq R_{\sigma}$. Furthemore, PSPACE-hardness for satisfiability is not a systematic feature of polymodal information 
logics (assuming NP $\neq$ PSPACE). For instance, the bimodal information logic containing a modal operator for indiscernibility and a modal operator for complementarity (see Section 1) has an NP-complete satisfiability problem [9]. Hence, a further analysis about the PSPACEcompleteness of NIL satisfiability shall certainly help understanding the complexity of other information logics and in a more general setting the computational complexity of numerous polymodal logics with interdependent modal connectives.

Acknowledgements: The author wish to thank Ewa Orłowska for her numerous suggestions on earlier versions of this work.

\section{References}

[1] Ph. Balbiani. Axiomatization of logics based on Kripke models with relative accessibility relations. In [26], pages 553-578, 1997.

[2] M. Baldoni, L. Giordano, and A. Martelli. A tableau calculus for multimodal logics and some (un)decidability results. In H. de Swart, editor, TABLEAUX-8, pages 44-59. LNAI 1397, SpringerVerlag, 1998.

[3] J. van Benthem. Modal logic and classical logic. Bibliopolis, 1985.

[4] P. Blackburn, M. de Rijke, and Y. Venema. Modal Logic. 2000. Forthcoming. Preprint available via http://turing.wins.uva.nl/ mdr/ on WWW.

[5] S. Cerrito and M. Cialdea Mayer. A polynomial translation of S4 into T and contractionfree tableaux for S4. Logic Journal of the IGPL, 5(2):287-300, 1997. Available via http://www3.oup.co.uk/igpl/contents/ on WWW.

[6] C.-C. Chen and I-P. Lin. The computational complexity of the satisfiability of modal Horn clauses for modal propositional logics. Theoretical Computer Science, 129:95-121, 1994.

[7] S. Demri. Sequent calculi for nominal tense logics: a step towards mechanization? In N. Murray, editor, TABLEAUX'99, pages 140-154. LNAI 1617, Springer-Verlag, 1999. Available via http://wwwleibniz.imag.fr/ATINF/ on WWW.

[8] S. Demri and B. Konikowska. Relative similarity logics are decidable: reduction to $\mathrm{FO}^{2}$ with equality. In JELIA'98, Dagsthul, Germany, pages 279-293. LNAI 1489, Springer-Verlag, 1998.

[9] S. Demri, E. Orłowska, and D. Vakarelov. Indiscernibility and complementarity relations in Pawlak's information systems. In J. Gerbrandy, M. Marx, M. de Rijke, and Y. Venema, editors, JFAK. Essays Dedicated to Johan van Benthem on the Occasion of his 50th Birthday., 1999. Available via http://www.illc.uva.nl/ j50/ on WWW.

[10] F. Donini, M. Lenzerini, D. Nardi, and W. Nutt. The complexity of concept languages. Information and Computation, 134:1-58, 1997.

[11] L. Fariñas del Cerro and E. Orłowska. DAL - A logic for data analysis. Theoretical Computer Science, 36:251-264, 1985. Corrigendum ibid, 47:345, 1986.

[12] M. Fischer and R. Ladner. Propositional dynamic logic of regular programs. Journal of Computer and System Sciences, 18:194-211, 1979. 
[13] M. Fitting. Proof methods for modal and intuitionistic logics. D. Reidel Publishing Co., 1983.

[14] M. Fitting. First-order modal tableaux. Journal of Automated Reasoning, 4:191-213, 1988.

[15] J. Halpern and Y. Moses. A guide to completeness and complexity for modal logics of knowledge and belief. Artificial Intelligence, 54:319-379, 1992.

[16] E. Hemaspaandra. Complexity transfer for modal logic (extended abstract). In Ninth Annual IEEE Symposium on Logic in Computer Science (LICS-9), pages 164-173, July 1994.

[17] E. Hemaspaandra. The price of universality. Notre Dame Journal of Formal Logic, 37(2):173-203, 1996.

[18] B. Konikowska. A logic for reasoning about relative similarity. Studia Logica, 58(1):185-226, 1997.

[19] M. Kracht. Power and weakness of the modal display calculus. In H. Wansing, editor, Proof theory of modal logic, pages 93-121. Kluwer, 1996.

[20] R. Ladner. The computational complexity of provability in systems of modal propositional logic. SIAM Journal of Computing, 6(3):467-480, September 1977.

[21] M. Marx. Complexity of modal logics of relations. Technical report, ILLC, 1997. To appear in Annals of Pure and Applied Logic. Available via http://turing.wins.uva.nl/ marx/ on WWW.

[22] F. Massacci. Single steps tableaux for modal logics. Journal of Automated Reasoning, 24(3):319-364, April 2000. Available via http://www.dis.uniroma1.it/ massacci on WWW.

[23] E. Orłowska. Introduction: What you always wanted to know about rough sets. In [26], pages 1-20, 1997.

[24] E. Orłowska and Z. Pawlak. Expressive power of knowledge representation system. Technical Report 432, Institute of Computer Science, Polish Academy of sciences, 1981.

[25] E. Orłowska and Z. Pawlak. Representation of nondeterministic information. Theoretical Computer Science, 29:27-39, 1984.

[26] E. Orłowska (ed.). Incomplete Information: Rough Set Analysis. Studies in Fuzziness and Soft Computing. Physica-Verlag, 1997.

[27] Ch. Papadimitriou. Computational Complexity. Addison-Wesley Publishing Company, 1994.

[28] Z. Pawlak. Information systems theoretical foundations. Information Systems, 6(3):205-218, 1981.

[29] V. Pratt. Models of program logics. In 20th IEEE Symposium on Foundations of Computer Science, pages $115-122,1979$.

[30] H. Sahlqvist. Completeness and correspondence in the first and second order semantics for modal logics. In S. Kanger, editor, 3rd Scandinavian Logic Symposium, Uppsala, Sweden, 1973, pages 110-143. North Holland, 1975.

[31] E. Spaan. The complexity of propositional tense logics. In M. de Rijke, editor, Diamonds and Defaults, pages 287-309. Kluwer Academic Publishers, Series Studies in Pure and Applied Intensional Logic, Volume 229, 1993.

[32] D. Vakarelov. Abstract characterization of some knowledge representation systems and the logic NIL of nondeterministic information. In Ph. Jorrand and V. Sgurev, editors, Artificial Intelligence: Methodology, Systems, Applications, pages 255-260. Elsevier Science Publishers B.V. (NorthHolland), 1987. 
[33] D. Vakarelov. Modal logics for knowledge representation systems. Theoretical Computer Science, 90:433-456, 1991.

[34] D. Vakarelov. Information systems, similarity and modal logics. In [26], pages 492-550, 1997.

[35] L. Vigano. Labelled Non-Classical Logics. Kluwer Academic Publishers, 2000. 